\title{
Classification of Single Normal and Alzheimer's Disease Individuals from Cortical Sources of Resting State EEG Rhythms
}

\begin{abstract}
Claudio Babiloni ${ }^{1,2 *}$, Antonio I. Triggiani ${ }^{3}$, Roberta Lizio ${ }^{1,2}$, Susanna Cordone ${ }^{1}$, Giacomo Tattoli ${ }^{4}$, Vitoantonio Bevilacqua ${ }^{4}$, Andrea Soricelli ${ }^{5,6}$, Raffaele Ferri ${ }^{7}$, Flavio Nobili ${ }^{8}$, Loreto Gesualdo ${ }^{9}$, José C. Millán-Calenti ${ }^{10}$, Ana Buján ${ }^{10}$, Rosanna Tortelli ${ }^{11}$, Valentina Cardinali ${ }^{11,12}$, Maria Rosaria Barulli ${ }^{13}$, Antonio Giannini ${ }^{14}$, Pantaleo Spagnolo ${ }^{15}$, Silvia Armenise ${ }^{16}$, Grazia Buenza ${ }^{11}$, Gaetano Scianatico ${ }^{13}$, Giancarlo Logroscino ${ }^{13,16}$, Giovanni B. Frisoni ${ }^{17,18}$ and Claudio del Percio ${ }^{5}$

1 Department of Physiology and Pharmacology "Vittorio Erspamer", University of Rome "La Sapienza", Rome, Italy, ${ }^{2}$ Department of Neuroscience, IRCCS San Raffaele Pisana, Rome, Italy, ${ }^{3}$ Department of Clinical and Experimental Medicine, University of Foggia, Foggia, Italy, ${ }^{4}$ Department of Electrical and Information Engineering, Polytechnic of Bari, Bari, Italy, ${ }^{5}$ Department of Integrated Imaging, IRCCS SDN - Istituto di Ricerca Diagnostica e Nucleare, Napoli, Italy, ${ }^{6}$ Department of Motor Sciences and Healthiness, University of Naples Parthenope, Naples, Italy, ${ }^{7}$ Department of Neurology, IRCCS Oasi Institute for Research on Mental Retardation and Brain Aging, Troina, Italy, ${ }^{8}$ Service of Clinical Neurophysiology (DiNOGMl; DipTeC), IRCCS Azienda Ospedaliera Universitaria San Martino - IST, Genoa, Italy, ${ }^{9}$ Dipartimento Emergenza e Trapianti d'Organi, University of Bari, Bari, Italy, ${ }^{10}$ Gerontology Research Group, Department of Medicine, Faculty of Health Sciences, University of A Coruña, A Coruña, Spain, " Department of Clinical Research in Neurology, University of Bari "Aldo Moro", Pia Fondazione Cardinale G. Panico, Lecce, Italy, ${ }^{12}$ Department of Basic Medical Sciences, Neurosciences and Sense Organs, University of Bari "Aldo Moro", Bari, Italy, ${ }^{13}$ Unit of Neurodegenerative Diseases, Department of Clinical Research in Neurology, University of Bari "Aldo Moro", Pia Fondazione Cardinale G. Panico, Lecce, Italy, ${ }^{14}$ Department of Imaging Division of Radiology, Hospital "Di Venere", Bari, Italy, ${ }^{15}$ Division of Neuroradiology, "F. Ferrari" Hospital, Lecce, Italy, ${ }^{16}$ Department of Basic Medical Sciences, Neuroscience and Sense Organs, University of Bari "Aldo Moro", Bari, Italy, ${ }^{17}$ Laboratory of Epidemiology, Neuroimaging and Telemedicine, IRCCS Centro "S. Giovanni di Dio-F.B.F.", Brescia, Italy,

${ }^{18}$ Memory Clinic and LANVIE - Laboratory of Neuroimaging of Aging, University Hospitals and University of Geneva, Geneva, Switzerland
\end{abstract}

claudio.babiloni@uniroma1.it

\section{Specialty section: \\ This article was submitted to \\ Neurodegeneration, \\ a section of the journal \\ Frontiers in Neuroscience}

Received: 29 August 2015 Accepted: 02 February 2016 Published: 23 February 2016

Citation:

Babiloni C, Triggiani Al, Lizio $R$, Cordone S, Tattoli G, Bevilacqua V,

Soricelli A, Ferri R, Nobili F, Gesualdo L, Millán-Calenti JC

Buján A, Tortelli R, Cardinali V, Barulli MR, Giannini A, Spagnolo P, Armenise S, Buenza G, Scianatico G,

Logroscino G, Frisoni GB and del Percio C (2016) Classification of Single Normal and Alzheimer's Disease Individuals from Cortical

Sources of Resting State EEG

Rhythms. Front. Neurosci. 10:47. doi: 10.3389/fnins.2016.00047
Previous studies have shown abnormal power and functional connectivity of resting state electroencephalographic (EEG) rhythms in groups of Alzheimer's disease (AD) compared to healthy elderly (Nold) subjects. Here we tested the best classification rate of 120 AD patients and 100 matched Nold subjects using EEG markers based on cortical sources of power and functional connectivity of these rhythms. EEG data were recorded during resting state eyes-closed condition. Exact low-resolution brain electromagnetic tomography (eLORETA) estimated the power and functional connectivity of cortical sources in frontal, central, parietal, occipital, temporal, and limbic regions. Delta $(2-4 \mathrm{~Hz})$, theta $(4-8 \mathrm{~Hz})$, alpha $1(8-10.5 \mathrm{~Hz})$, alpha $2(10.5-13 \mathrm{~Hz})$, beta $1(13-20 \mathrm{~Hz})$, beta $2(20-30 \mathrm{~Hz})$, and gamma $(30-40 \mathrm{~Hz})$ were the frequency bands of interest. The classification rates of interest were those with an area under the receiver operating characteristic curve (AUROC) higher than 0.7 as a threshold for a moderate classification rate (i.e., 70\%). Results showed that the following EEG markers overcame this threshold: (i) central, parietal, occipital, temporal, and limbic delta/alpha 1 current density; (ii) central, parietal, occipital temporal, and limbic delta/alpha 2 current density; (iii) frontal theta/alpha 1 current density; (iv) occipital delta/alpha 1 inter-hemispherical connectivity; (v) occipital-temporal theta/alpha 1 right 
and left intra-hemispherical connectivity; and (vi) parietal-limbic alpha 1 right intrahemispherical connectivity. Occipital delta/alpha 1 current density showed the best classification rate (sensitivity of $73.3 \%$, specificity of $78 \%$, accuracy of $75.5 \%$, and AUROC of $82 \%)$. These results suggest that EEG source markers can classify Nold and $A D$ individuals with a moderate classification rate higher than $80 \%$.

Keywords: Alzheimer's disease (AD), electroencephalography (EEG), exact low-resolution brain electromagnetic tomography (eLORETA), spectral coherence, lagged linear connectivity, area under the receiver operating characteristic curve (AUROC), delta rhythms, alpha rhythms

\section{INTRODUCTION}

Alzheimer's disease (AD) is the most prevalent brain neurodegenerative disorder progressing to severe cognitive impairment and loss of autonomy (i.e., dementia) in older people (Braak and Braak, 1995; Jelic et al., 1998; Price, 2000; Leite et al., 2004; Glodzik-Sobanska et al., 2005; Kang et al., 2015).

Criteria for clinical diagnosis of $\mathrm{AD}$ were proposed in 1984 (McKhann et al., 1984) by the National Institute of Neurological and Communicative Disorders and Stroke (NINCDS) and by the Alzheimer's Disease and Related Disorders Association (ADRDA). According to these criteria, the final diagnosis of definite $\mathrm{AD}$ needed histopathologic confirmation (i.e., microscopic examination of brain tissue) in autopsy or biopsy.

In the past years, the International Working Group (IWG) and the US National Institute on Aging-Alzheimer's Association (NIA-AA) have proposed an algorithm for the diagnosis of $\mathrm{AD}$ at the preclinical (before any objective clinical manifestation), prodromal (with objective mild cognitive impairment [MCI] but with autonomy substantially preserved), and overt dementia stages, based on in vivo fluid and neuroimaging biomarkers as well as clinical phenotypes of the disease (Förstl and Kurz, 1999; Dubois et al., 2014). The neuroimaging biomarkers included brain hypometabolism, as revealed by fluorodeoxyglucose positron emission tomography (FDG-PET), brain amyloid load, as measured by ligand PET, and maps of brain atrophy and abnormalities of structural brain connectivity, as revealed by magnetic resonance imaging (MRI).

The mentioned PET and MRI methodologies capture several processes of $\mathrm{AD}$, but cannot be always used due to the limited availability of the instrumental resources, costs, invasiveness, or radiation exposure (e.g., PET). These limitations are problematic especially for serial recordings over time. In contrast, electroencephalographic (EEG) recordings in awake resting state condition represent an ideal low-cost and noninvasive methodology for clinical applications. Indeed, EEG recording has a high temporal resolution (milliseconds) that provides an optimal investigational tool for the emerging features of brain physiology, namely its oscillatory nature (Schroeter et al., 2009; Babiloni et al., 2013a). Indeed, EEG rhythms are the most important features of the collective behavior of brain neural populations and are very relevant for human cognition. Furthermore, EEG procedures are widely available in any country. They are well-tolerated by patients and are not affected by the task difficulty. Moreover, they can be repeated over time without habituation effects. As an important methodological limitation, recorded EEG data require an expert manual verification of the EEG epochs free from artifacts, and commercial pieces of software do not provide immediate statistical indexes of abnormalities of EEG markers with respect to a normative database.

Previous studies in $\mathrm{AD}$ patients and elderly subjects with amnesic MCI have shown that resting state eyes-closed EEG rhythms may be promising markers for a neurophysiological evaluation of disease status. Noteworthy, these markers do not have a diagnostic value, as EEG rhythms do not directly reflect the pathophysiological markers of AD. Rather, they may be part of the "topographical markers" of AD according to the definition by Dubois et al. (2014). These topographic markers are not specific for $\mathrm{AD}$ but can provide an index of the extent to which $\mathrm{AD}$ subjects show the abnormal structure and/or function of the brain across time. In this framework of topographic markers, the resting state EEG rhythms may reveal abnormalities of basic neurophysiological mechanisms underlying vigilance and cognition in $\mathrm{AD}$ subjects. When compared to groups of normal elderly (Nold) subjects, $\mathrm{AD}$ groups are characterized by high power of widespread delta $(<4 \mathrm{~Hz})$ and theta $(4-7 \mathrm{~Hz})$ rhythms, as well as by low power of posterior alpha $(8-12 \mathrm{~Hz})$ and/or beta (13-20 Hz) rhythms (Dierks et al., 1993, 2000; Huang et al., 2000; Ponomareva et al., 2003; Jeong, 2004). Similarly, MCI subjects display increased theta power (Grunwald et al., 2001) as well as decreased alpha power (Jelic et al., 1998, 2000; Huang et al., 2000; Grunwald et al., 2001). In line with the "transition" hypothesis, power of resting state EEG alpha rhythms is high in Nold subjects, intermediate in MCI subjects, and low in AD patients at the group level (Elmstáhl and Rosén, 1997; Huang et al., 2000; Jelic et al., 2000; Jeong, 2004).

Markers of resting state eyes-closed EEG rhythms have unveiled abnormalities of neurophysiological mechanisms in $\mathrm{AD}$ patients not only at the group but also at the individual level. A correct classification of up to $90 \%$ of success between Nold and $\mathrm{AD}$ individuals by using EEG markers as an input to artificial neural network (ANN) classifiers with the leave-one-out crossvalidation method has been previously reported (Anderer et al., 1994; Pritchard et al., 1994). Similar results were obtained in the correct classification not only between $\mathrm{AD}$ and Nold subjects but also between Nold and MCI subjects (Adler et al., 2003; Brassen et al., 2004). Unfortunately, those results suffered from the use of a small number of subjects. Furthermore, Lehmann et al. (2007) used EEG markers as an input to random forest procedures and obtained a correct classification between $\mathrm{AD}$ and Nold individuals of about $82 \%$. This classification rate improved 
to about $89 \%$ using ANN (Lehmann et al., 2007). Buscema and colleagues reached about $92 \%$ of success in the classification of $\mathrm{AD}$ and $\mathrm{MCI}$ individuals using EEG markers as an input to ANNs (Buscema et al., 2007), while Dauwels and colleagues reached classification rates of 83 and $88 \%$ for pre-dementia and mild AD, respectively (Dauwels et al., 2009). The same research group has also performed a recent study (Dauwels et al., 2010) comparing the classification rate between Nold and MCI individuals based on a large number of EEG markers, such as partial directed coherence, directed transfer function (DTF), full frequency DTF (ffDTF), different types of entropy measures, state space-based estimators, information-theoretic measures including Mutual Information (a measure closely related to Synchronization Likelihood), and different types of divergence and stochastic event synchrony (SES). Results revealed that most of these EEG markers globally indicated decreased functional and less effective brain connectivity in MCI patients with respect to Nold subjects. However, only two EEG markers yielded significant results in the classification of Nold and MCI individuals, namely SES and ffDTF, with a correct classification of about $83 \%$ using linear and quadratic discriminant analysis with leave-one-out crossvalidation (Dauwels et al., 2010).

Previous studies have shown interesting results on markers of $\mathrm{AD}$ based on magnetoencephalographic (MEG) counterpart of resting state EEG rhythms. Specifically, Gómez et al. (2009) analyzed the resting state MEG rhythms in $20 \mathrm{AD}$ patients and 21 Nold subjects to test the ability of the dimensional complexity of MEG signals (as measured by Higuchi's fractal dimension) to classify those individuals correctly. Results showed that MEG signals had lower complexity values in $\mathrm{AD}$ patients than in Nold subjects. Furthermore, the accuracy of $87.8 \%$ ( $80 \%$ sensitivity; $95.2 \%$ specificity) in the classification of Nold and AD subjects was obtained by computation of receiver operating characteristic (ROC) curves. Moreover, Fernández et al. (2013) demonstrated that source current density values of MEG delta rhythms in posterior parietal, occipital, pre-rolandic, and precuneus cortices distinguished reliably between MCI patients, $\mathrm{AD}$ patients with different severity scores, and Nold subjects. Based on these results, the Authors proposed MEG delta markers as a promising candidate for the detection of $\mathrm{AD}$ patients in clinical practice.

The present research group has been investigating markers of resting state eyes-closed EEG rhythms in MCI, $\mathrm{AD}$, and control subjects in the framework of the "BRAINON" program (www.brainon.eu). In previous studies of this program, cortical sources of resting state eyes-closed EEG rhythms were estimated and compared between groups of $\mathrm{MCI}, \mathrm{AD}$, and control subjects, in order to enhance spatial information content of scalp-recorded EEG data and to unveil topography of EEG abnormalities associated with $\mathrm{AD}$ from prodromal to overt clinical stages (Babiloni et al., 2004a, 2008, 2011a,b,c, 2013a,b,c). For this purpose, low-resolution brain electromagnetic tomography (LORETA) was used (Pascual-Marqui et al., 1994). Overall, it was found that occipital, parietal, and temporal cortical sources of delta and alpha rhythms showed abnormal activity in groups of $\mathrm{AD}$ subjects when compared to control groups. Furthermore, this abnormal activity was related to markers of hippocampus atrophy, cortical atrophy, and vascular lesions of white matter. In the same line, when compared to control groups, groups of AD subjects showed abnormal functional coupling of these EEG rhythms as revealed by spectral coherence and other techniques of functional connectivity (Babiloni et al., 2004b, 2006b, 2008, 2009a, 2010).

As a new milestone of the "BRAINON" program, the current study tested several resting state EEG markers as ability to classify correctly AD individuals with dementia and Nold individuals. These EEG markers were obtained by the estimation of activity and functional connectivity of the cortical sources of resting state eyes-closed EEG rhythms by the use of exact LORETA (eLORETA; Pascual-Marqui, 2007a). The EEG markers of interest were used as discriminant variables for the computation of the rate of classification between single Nold and $\mathrm{AD}$ individuals.

\section{MATERIALS AND METHODS \\ Subjects and Diagnostic Criteria}

This study involved 120 AD and 100 Nold individuals, carefully matched for age, gender, and years of education. They were selected blindly to the features of EEG data, to avoid any logical circularity or bias in the results.

Committees of local institutional ethics approved the recording and analysis of EEG data for scientific purposes. All experiments were performed with the informed consent of each participant or caregiver, in line with the Code of Ethics of the World Medical Association (Declaration of Helsinki). The inclusion criteria for AD individuals comprised the diagnosis of mild to moderate $\mathrm{AD}$ according to NINCDSADRDA (McKhann et al., 1984) and DSM-IV guidelines. Furthermore, these individuals had to have received medical, neurological, neuropsychological, and psychiatric assessments including MMSE (Folstein et al., 1975), Clinical Dementia Rating (CDR; Hughes et al., 1982), geriatric depression scale (GDS; Yesavage et al., 1982), and Instrumental Activities of Daily Living scale (IADL; Lawton and Brody, 1969). Exclusion criteria included scores in the MMSE lower than 27 in the Nold subjects and higher than 24 in the AD subjects according to Alzheimer's Disease Neuroimaging Initiative, ADNI (http://adni.loni.usc.edu). Besides, exclusion criteria comprised any kind of evidence of other forms or causes of dementia such as frontotemporal dementia (The Lund Manchester Groups, 1994), vascular dementia, (NINDS-AIREN criteria; Román et al., 1993), Parkinson disease, Lewy body dementia (McKeith et al., 2005), metabolic syndrome, nutritional deficits, tumors, etc. The Nold subjects had no history of neurological or major psychiatric disorders. They underwent medical, neurological, and psychiatric assessments including MMSE and GDS, to exclude actual neurocognitive disorders and major psychiatric symptoms (including abuse of substances).

Table 1 reports information about personal and clinical characteristics of the Nold and AD subjects of the present study. An independent $t$-test evaluated the presence or absence of statistically significant differences ( $p<0.05$, one-tailed) between the two groups (i.e., Nold and AD patients) for the age, education, 
TABLE 1 | Demographic and clinical data of normal elderly (Nold) subjects and Alzheimer's disease (AD) patients.

\begin{tabular}{lcccr}
\hline & $\begin{array}{c}\text { Gender } \\
\text { (Female/Male) }\end{array}$ & $\begin{array}{c}\text { Age } \\
\text { (years) }\end{array}$ & \multicolumn{1}{c}{$\begin{array}{c}\text { Education } \\
\text { (years) }\end{array}$} & \multicolumn{1}{c}{$\begin{array}{l}\text { MMSE } \\
\text { (score) }\end{array}$} \\
\hline Nold $(n=100)$ & $62 / 38$ & $69 \pm 0.9$ SE & $9.7 \pm 0.4$ SE & $28.8 \pm 0.1 \mathrm{SE}$ \\
$\operatorname{AD~}(n=120)$ & $78 / 42$ & $69.8 \pm 0.7$ SE & $9.2 \pm 0.4$ SE & $19 \pm 0.3 \mathrm{SE}$
\end{tabular}

MMSE, Mini Mental State Evaluation.

individual alpha frequency (IAF; see below for a description of this index), and MMSE score. As expected, a statistically significant difference was found for the MMSE score $(p<0.0001$; higher MMSE score in the Nold than in the AD group) and for the IAF ( $p<0.0001$; higher IAF in the Nold than in the AD group). On the contrary, no statistically significant difference was found for age, gender, and education $(p>0.05)$.

\section{EEG Recordings}

All subjects were kindly asked to stay relaxed at eyes closed and not to move or talk. EEG data were recorded (bandpass: 0.01-100 Hz; EB-Neuro Be-light $\odot$, Firenze, Italy) during resting state eyes-closed condition from 19 scalp electrodes positioned over the whole head according to the 10-20 System (i.e., Fp1, Fp2, F7, F3, Fz,F4, F8, T3, C3, Cz, C4, T4, T5, P3, Pz, P4, T6, O1, O2; Figure 1). Linked earlobe reference electrode was appreciated but not mandatory to respect the methodological facilities and standard internal protocols of the clinical recording units. A ground electrode was located between the $\mathrm{AFz}$ and $\mathrm{Fz}$ electrodes. Electrode impedance was kept below 5 Kohm. All recorded artifact-free EEG data were off-line rereferenced to common average to harmonize the EEG data collected with different reference electrodes. The horizontal and vertical electro-oculographic activities $(0.3-70 \mathrm{~Hz}$ bandpass) were simultaneously recorded to monitor eye movements. All data were digitalized in continuous recording mode (about 5 min of EEG; 128-256 Hz sampling rate set to avoid aliasing).

\section{Preliminary EEG Data Analysis}

The EEG data were segmented in consecutive epochs of $2 \mathrm{~s}$ and were analyzed off-line. The epochs affected by any artifacts (ocular, muscular, instrumental) were preliminarily identified by an automatic computerized procedure. Two independent experimenters manually confirmed the artifact-free EEG epochs that were accepted for further analysis. Particular attention was paid to exclude EEG epochs with signs of drowsiness or pre-sleep stages.

\section{Spectral Analysis of EEG Rhythms}

The power spectral density of the EEG rhythms was computed using an FFT analysis (Welch method, Hanning windowing function, no phase shift) with $0.5 \mathrm{~Hz}$ of frequency resolution. The following standard frequency bands of interest were considered, in line with previous relevant EEG studies (Besthorn et al., 1997; Chiaramonti et al., 1997; Babiloni et al., 2005, 2006b, 2011b, 2013c): delta $(2-4 \mathrm{~Hz})$, theta $(4-8 \mathrm{~Hz})$, alpha $1(8-10.5 \mathrm{~Hz})$, alpha $2(10.5-13 \mathrm{~Hz})$, beta $1(13-20 \mathrm{~Hz})$, beta $2(20-30 \mathrm{~Hz})$, and gamma

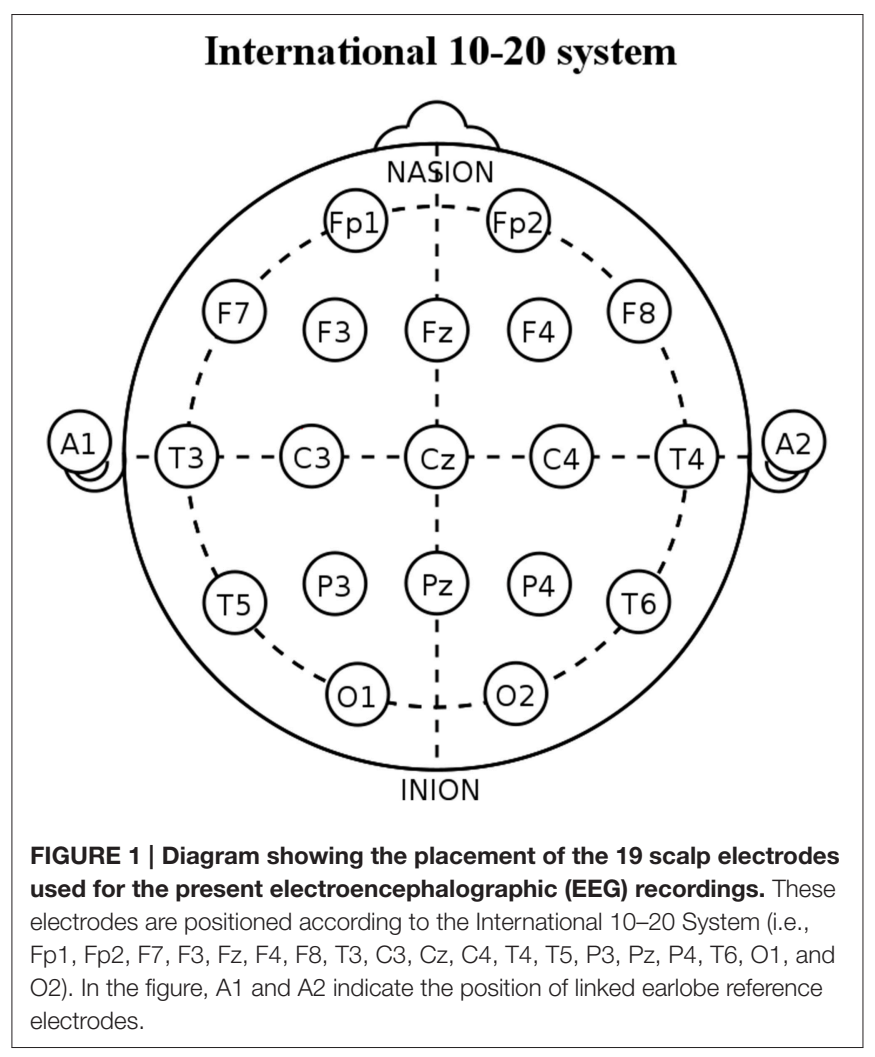

$(30-40 \mathrm{~Hz})$. These frequency bands were computed sharing the frequency bin by two contiguous bands, as a widely accepted procedure (Jelic et al., 1996; Besthorn et al., 1997) that fits the theoretical consideration that near EEG rhythms may overlap at their frequency borders (Klimesch et al., 1996; Klimesch, 1999; Babiloni et al., 2005, 2010). Individual alpha frequency (IAF) peak was defined as the frequency associated with the strongest EEG power at the extended alpha range of $7-14 \mathrm{~Hz}$ (Klimesch, 1999).

\section{Cortical Source of EEG Rhythms as Computed by eLORETA}

As mentioned above, eLORETA estimated the activity of the cortical sources of EEG rhythms (Pascual-Marqui, 2007a). eLORETA is an improvement over previous well-known techniques called LORETA (Pascual-Marqui et al., 1994) and the standardized LORETA (sLORETA; Pascual-Marqui, 2002). In simulation studies, both sLORETA and eLORETA showed low spatial resolution but zero localization error in the presence of measurement and biological noise (Pascual-Marqui, 2002, 2007a). Furthermore, it was reported a better source location by eLORETA than sLORETA (Canuet et al., 2011).

eLORETA uses a realistic head model (Fuchs et al., 2002) using the MNI152 template (Mazziotta et al., 2001), with a threedimensional solution space restricted to cortical gray matter and electrode coordinates provided by Jurcak (Jurcak et al., 2007). The intracerebral volume is partitioned in 6239 voxels with $5 \mathrm{~mm}$ spatial resolution. Thus, eLORETA images obtained from resting 
TABLE 2 | Regions of interest (ROIs) for the estimation of the cortical sources of resting state eyes-closed electroencephalographic (EEG) rhythms by exact low-resolution brain electromagnetic tomography (eLORETA) software.

\begin{tabular}{ll} 
Brodmann areas into the regions of interest (ROIs) \\
\hline Frontal & $8,9,10,11,44,45,46,47$ \\
Central & $1,2,3,4,6$ \\
Parietal & $5,7,30,39,40,43$ \\
Temporal & $20,21,22,37,38,41,42$ \\
Occipital & $17,18,19$ \\
Limbic & $31,32,33,34,35,36$
\end{tabular}

Any $R O I$ is defined by some Brodmann areas within the cortical source space of the eLORETA software.

state EEG data represent the electrical activity at each voxel in the neuroanatomic Montreal Neurological Institute (MNI) space as the exact magnitude of the estimate current density. Anatomical labels as Brodmann areas are also reported using MNI space, with adaptation to Talairach space (Brett et al., 2002).

eLORETA cortical source solutions did estimate current density values at $\mathrm{x}, \mathrm{y}$, and $\mathrm{z}$ vectors of any brain voxel able to predict EEG spectral power density at all scalp electrodes. Among the infinite solutions to the EEG inverse problem, a regularization procedure selected the maximally smoothed solution at the cortical source level of the eLORETA head model. Afterwards, this solution was normalized by the computation of the eLORETA current density at each voxel (as the mean of the $\mathrm{x}, \mathrm{y}$, and $\mathrm{z}$ vectors) with current density value averaged across all frequencies $(0.5-45 \mathrm{~Hz})$ and 6239 voxels of the brain volume. For this reason, normalized eLORETA solutions are reported by an arbitrary unit scale in which " 1 " means equal to the average value of eLORETA current density computed across all frequencies and voxels. The general procedure typical fits EEG power density in a Gaussian distribution and reduces inter-subject variability (Leuchter et al., 1993).

In line with the general low spatial resolution of the present EEG methodological approach (i.e., 19 scalp electrodes), the eLORETA solutions were averaged across all voxels in a given cortical macro region of interest (ROI). Frontal, central, parietal, occipital, temporal, and limbic ROIs were considered. Table 2 reports the Brodmann areas (BAs) included in these ROIs. The eLORETA cortical sources at these ROIs and all frequency bands of interest were used as a first set of EEG markers for the present classification purposes.

\section{EEG Lagged Linear Connectivity by eLORETA}

Functional connectivity between two regions has previously been defined as the non-linear and linear dependence, as for example, lagged non-linear and linear coherence of intra-cortical EEGsource estimates (Pascual-Marqui, 2007b). When computed in the cortical source space, the inherent low spatial resolution of the EEG tomography enters high phase synchronization and zero-lag coherence (Pascual-Marqui, 2007b). Activity at any cortical area is observed instantaneously (zero-lag) by all scalp electrodes. Instantaneous coherence between two cortical sources might also be found when a third source has an impact on two other brain sources even whether the two paired sources do not influence each other or as there is activity at reference electrode in coherence analysis. For this reason, only the lagged coherence contribution of these measures should be considered for more save neurophysiological considerations. In this line, PascualMarqui (2007b) proposed the solution to remove the zerolag instantaneous interactions and to compute coherence using the residual, corrected time series. Furthermore, the proposed solution included measures of dependence among multivariate EEG time series (Pascual-Marqui, 2007c). This procedure of functional connectivity was called lagged linear connectivity (LLC) and was implemented to make it available a freeware in the eLORETA package (Pascual-Marqui et al., 2011). In the present study, we used that freeware (Pascual-Marqui et al., 2011) to test the classification accuracy of EEG markers of functional connectivity among EEG cortical sources in Nold and AD individuals.

For each subject and frequency band of interest (i.e., delta, theta, alpha 1 , alpha 2 , beta 1 , beta 2 , and gamma), the LLC was computed for six ROIs (i.e., frontal, central, parietal, occipital, temporal, and limbic). For the inter-hemispherical analysis, the LLC estimates were calculated between all voxels of the mentioned ROIs of each hemisphere with the corresponding ones of the other hemisphere. The LLC solutions for all voxels of a given pair of ROIs were averaged. For the intra-hemispherical analysis, the LLC estimates were computed for all voxels of a particular ROI with all voxels of another ROI of the same hemisphere. The LLC solutions for all voxels of a given pair of ROIs were averaged. This operation was repeated for the left/and the right hemisphere.

\section{Statistical Analysis of the eLORETA Computations}

To test the working hypotheses related to EEG power activity and functional connectivity as revealed by eLORETA solutions, two statistical sessions were performed by the commercial tool STATISTICA 10 (StatSoft Inc., www.statsoft.com).

The first statistical session tested the hypothesis that mean current density in the ROIs would differ between the Nold and AD groups. To this aim, a Three-way ANOVA was computed using regional normalized eLORETA solutions (normalized current density at all voxels of a given ROI) as a dependent variable $(p<0.05)$. The ANOVA factors were Group (Nold, $\mathrm{AD}$ ), Band (delta, theta, alpha 1, alpha 2, beta 1, beta 2, and gamma), and ROI (frontal, central, parietal, occipital, temporal, and limbic). Subjects' age, education, IAF, and gender were used as covariates. Mauchly's test evaluated the sphericity assumption. The degrees of freedom were corrected by Greenhouse-Geisser procedure when appropriate. Duncan test was used for posthoc comparisons ( $p<0.05$, one-tailed). Of note, the present study was not focused on the differences of EEG cortical sources between the Nold and the AD group. That focus would have required a conservative post-hoc test using a correction for multiple univariate comparisons at $p<0.05$. Rather, this study 
tested the ability of EEG source markers to classify Nold and AD individuals. In this line, ANOVA and post-hoc tests were used merely to reduce the amount of EEG markers to be used for the calculation of that ability. For this reason, we used the liberal onetailed analysis rather than a conservative correction for multiple univariate statistical comparisons.

The second statistical session tested the hypothesis that the mean functional connectivity among ROIs would differ between the Nold and AD groups. Different Three-way ANOVAs were performed using the LLC calculated with eLORETA as a dependent variable $(p<0.05)$. The first ANOVA tested the mean differences of inter-hemispherical LLC between the two groups. The ANOVA factors were Group (Nold, AD), Band (delta, theta, alpha 1 , alpha 2 , beta 1 , beta 2 , and gamma), and ROI pairs (frontal left-frontal right, central left-central right, parietal left-parietal right, occipital left-occipital right, temporal left-temporal right, and limbic left-limbic right). The second ANOVA tested the mean differences of left intra-hemispherical LLC between the two groups. The ANOVA factors were Group (Nold, AD), Band (delta, theta, alpha 1, alpha 2, beta 1, beta 2, and gamma), and ROI pairs (frontal-central, frontal-parietal, frontaloccipital, frontal-temporal, frontal-limbic, central-parietal, central-occipital, central, temporal, central-limbic, parietaloccipital, parietal-temporal, parietal-limbic, occipital-temporal, occipital-limbic, and temporal-limbic). The third ANOVA had the same design for the right hemisphere. In the three ANOVAs, subjects' age, education, IAF, and gender were used as covariates. Mauchly's test evaluated the sphericity assumption. The degrees of freedom were corrected by Greenhouse-Geisser procedure when appropriate. Duncan test was used for post-hoc comparisons ( $p<0.05$, one-tailed).

\section{Accuracy of the EEG Markers in the Discrimination between Nold and AD Individuals}

The third statistical session tested the ability of the EEG markers (i.e., source current density and LLC) to classify single Nold and $\mathrm{AD}$ individuals. To this aim, the EEG markers showing statistical differences between the Nold and the AD group in those two statistical sessions were used as input variables for the third statistical session. The classification rate was computed by the analysis of the receiver operating characteristic curve (ROC; DeLong et al., 1988). This analysis was performed by Matlab 2010b software (Mathworks Inc., Natick, MA, USA). The following indexes measured the classification rate of the above binary classification (i.e., Nold vs. AD):

1) Sensitivity defined as the rate of the $\mathrm{AD}$ subjects correctly classified as $\mathrm{AD}$ (i.e., true positive rate);

2) Specificity defined as the rate of the Nold (control) subjects correctly classified as Nold (i.e., true negative rate);

3) Accuracy defined as the mean between the sensitivity and specificity;

4) The area under the ROC curve (AUROC) expressed as a percentage. Noteworthy, the EEG markers of interest were those showing an AUROC higher than an arbitrary threshold of $70 \%$ (i.e., the threshold of the so-called "moderate" classification rate, DeLong et al., 1988).

\section{Evaluation of the Relationship between the EEG Markers and Relevant Variables of AD}

Based on the values of the EEG markers, we defined AD individuals with the EEG marker "positive" (i.e., $\mathrm{AD}+$ ) as those having marker values equal or higher than the mean plus one standard deviation (SD) of the marker value in the Nold reference population. This $\mathrm{AD}+$ condition is expected to indicate abnormal values of the EEG marker in AD individuals as compared to the Nold group. In this line, the $\mathrm{AD}$ individuals with the EEG marker "negative" (i.e., $\mathrm{AD}-$ ) were those having marker values within the mean plus one $\mathrm{SD}$ of the marker value in the Nold reference population. To test the hypothesis that the $\mathrm{AD}+$ condition is related to relevant disease variables in $\mathrm{AD}$ patients, the MMSE score was compared between the AD+ subgroup and the $\mathrm{AD}-$ subgroup by independent $t$-test ( $p<0.05$, one tailed).

In the same line, we also hypothesized that the $\mathrm{AD}+$ condition is related to more abnormal brain structure in $\mathrm{AD}$ patients. To test this hypothesis, cortical gray matter (GM), subcortical white matter (WM), and cerebrospinal fluid (CSF) normalized volumes were estimated in 39 of the $120 \mathrm{AD}$ patients (those having T1- and T2-weigthed structural magnetic resonance imagingMRI available). These MRIs had been acquired following standard research settings mostly by $1.5 \mathrm{~T}$ scanners. The MRI scans were visually inspected to verify the absence of structural abnormalities or technical artifacts. Centralized MRI data analysis was performed by MATLAB 7.1 (MathWorks, Natick, MA) and SPM8 (Wellcome Dept. Cogn. Neurol., London; http://www.fil.ion.ucl.ac.uk/spm).

Specifically, the processing of the MRI data was as follows: the native MRI data of each patient were partitioned into GM, WM, and CSF compartments and spatially normalized to fit a standard labeled template, obtaining the transformation matrix (Ashburner and Friston, 1997). This labeled template was based on averaged high-resolution MRIs acquired from 24 subjects, comprising anatomic channels (T1, T2, and proton density weighted), tissue channels (CSF probability, GM probability, WM probability, and tissue labels), and the LPBA40 cortical parcellation map, based on the LONI Probabilistic Brain Atlas of 40 subjects (Shattuck et al., 2008) and identifying 56 brain structures. Second, the inverse of the transformation matrix was used to map the 56 atlas brain structures to the partitioned GM compartment, enabling volume quantification of each structure. Thirdly, a homemade MATLAB script was used to calculate the volume of cortical GM, and of the entire (cortical and subcortical) GM, WM, and CSF compartments. The normalized volumes of cortical GM, and WM and CSF compartments were obtained dividing the volume of each compartment by the total (GM, $\mathrm{WM}$, and CSF) volume. Of note, the above procedure seemed to be more appropriate than voxel-based morphometry (Good et al., 2001) for the analysis of the relationship between lowresolution (LORETA) EEG source estimates and MRI markers. Indeed, voxel-based morphometry is based on an intrinsically high-resolution voxel-by-voxel approach. 


\section{STATISTICAL ANOVA INTERACTION AMONG GROUP, BAND, ROI}

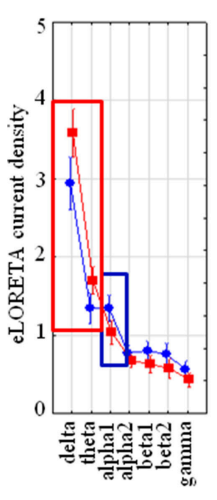

frontal

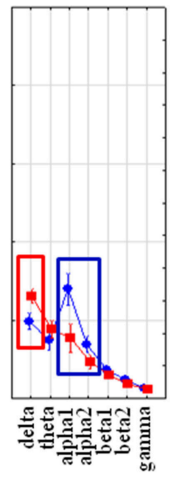

central

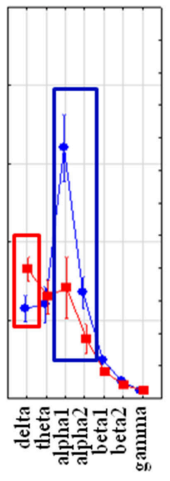

parietal

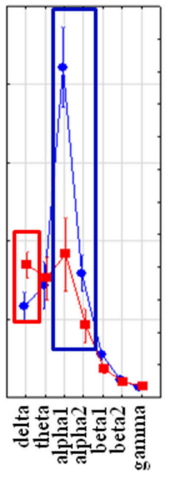

occipital

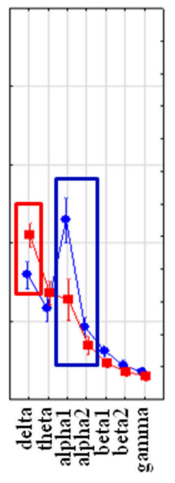

temporal

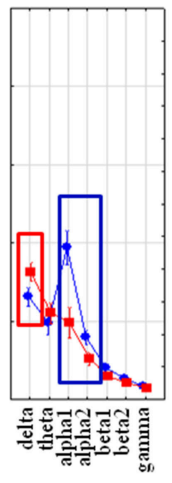

limbic

$$
\mp \text { Nold } \leftrightarrows \mathrm{AD}
$$

$\mathrm{AD}<$ Nold $(\mathrm{p}<0.05)$

$A D>\operatorname{Nold}(p<0.05)$

FIGURE 2 | Diagram showing the grand average of regional normalized exact low-resolution brain electromagnetic tomography (eLORETA) solutions (i.e., source activity) relative to a statistically significant ANOVA interaction $\left[F_{(30,6540)}=18.727, p<0.0001\right]$ among the factors Group (AD, Nold), Band (delta, theta, alpha 1, alpha 2, beta 1, beta 2, gamma), and ROI (central, frontal, parietal, occipital, temporal, limbic). Subjects' age, education, IAF, and gender were used as covariates. Legend: the rectangles indicate the cortical regions and frequency bands in which source activity presented the statistically significant LORETA pattern of source activity Nold $\neq \mathrm{AD}$ (Duncan test, $p<0.05$ ).

TABLE 3 | $P$-values (Duncan post-hoc) of the ANOVA related to the comparisons of source activity showing a statistically significant interaction $\left[F_{(30,6540)}=18.727, p<0.0001\right]$ among the factors Group (AD, Nold), Band (delta, theta, alpha 1, alpha 2, beta 1, beta 2, gamma), and ROI (central, frontal, parietal, occipital, temporal, limbic) and $p$-values of the $t$-tests for each composite EEG marker.

\begin{tabular}{|c|c|c|c|c|c|c|}
\hline \multicolumn{7}{|c|}{$p$-values of the ANOVA and $t$-test } \\
\hline & Frontal & Central & Parietal & Occipital & Temporal & Limbic \\
\hline Delta & 0.00001 & 0.001 & 0.000002 & 0.000002 & 0.000005 & 0.001 \\
\hline Theta & 0.0004 & n.s. & n.s. & n.s. & n.s. & n.s. \\
\hline Alpha1 & 0.002 & 0.000001 & 0.000002 & 0.000005 & 0.000002 & 0.000001 \\
\hline Alpha2 & n.s. & 0.03 & 0.000001 & 0.000001 & 0.02 & 0.01 \\
\hline Delta/Alpha1 & 0.00004 & 0.000004 & 0.0000001 & 0.0000001 & 0.0000001 & 0.000001 \\
\hline Theta/Alpha1 & 0.0000001 & - & - & - & - & - \\
\hline Delta/Alpha2 & - & 0.00007 & 0.0000001 & 0.0000001 & 0.0000001 & 0.0000001 \\
\hline
\end{tabular}

The comparison of the GM, WM, and CSF normalized volumes in the $\mathrm{AD}+$ subgroup and in the $\mathrm{AD}-$ subgroup was performed by a Two-way ANOVA using the normalized volume as a dependent variable $(p<0.05)$. The ANOVA factors were Group (AD+, $\mathrm{AD}-$ ) and Brain volume (GM, WM, and CSF). Mauchly's test evaluated the sphericity assumption. Correction of the degrees of freedom was made with Greenhouse-Geisser procedure when appropriate. The Duncan test was used for post-hoc comparisons ( $p<0.05$, one tailed).

All the analysis performed to test the relationship between the EEG markers and relevant variables of AD (MMSE scores, GM, WM, and CSF normalized volumes) constituted the fourth statistical session.

\section{RESULTS}

\section{Results of the First Statistical Session: Source Current Density of EEG Rhythms}

Figure 2 shows the grand average across subjects of the activity of regional EEG cortical sources relative to a statistically significant ANOVA interaction $\left[F_{(30,6540)}=18.727, p<0.0001\right]$ among the factors Group (Nold, AD), ROI (frontal, central, parietal, occipital, temporal, and limbic), and Band (delta, theta, alpha 1, alpha 2, beta 1 , beta 2, gamma). Subjects' age, education, IAF, and gender were used as covariates. Planned post-hoc testing unveiled the following statistically significant results (see Table 3). Compared to the Nold group, the AD group showed a 


\section{STATISTICAL ANOVA INTERACTION AMONG GROUP, BAND, ROI PAIRS}

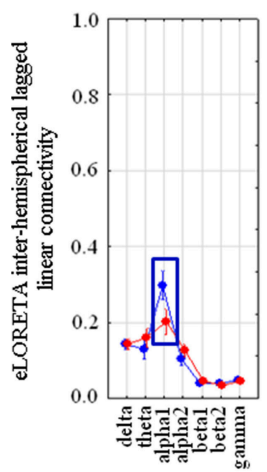

frontal

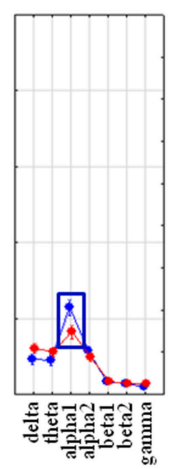

central

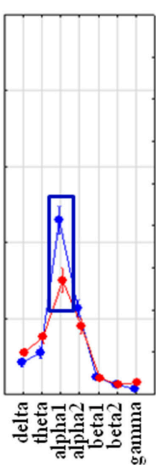

parietal

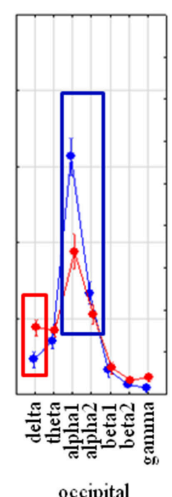

occipital

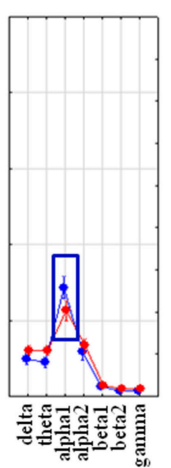

temporal

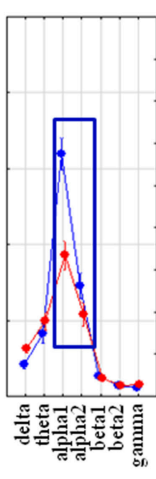

limbic AD

$\mathrm{AD}<\operatorname{Nold}(\mathrm{p}<0.05)$

$\mathrm{AD}>\operatorname{Nold}(\mathrm{p}<0.05)$

FIGURE 3 | Diagram showing the grand average of the EEG inter-hemispherical lagged linear connectivity computed between left and right hemispheres in the six regions of interest (ROI). These values refer a statistically significant ANOVA interaction $\left[F_{(30,6540)}=4.8771, p<0.0001\right]$ among the factors Group (Nold, and MCl), Band (delta, theta, alpha 1, alpha 2, beta 1, beta 2, gamma), and ROI pairs (frontal left-frontal right, central left-central right, parietal left-parietal right, occipital left-occipital right, temporal left-temporal right, and limbic left-limbic right). Subjects' age, education, IAF, and gender were used as covariates. Legend: the rectangles indicate the ROls and frequency bands in which connectivity values presented the pattern Nold $\neq$ AD (Duncan test, $p<0.05$ ).

TABLE 4 | p-values (Duncan post-hoc) of the ANOVA related to the comparisons of inter-hemispherical lagged linear connectivity showing a statistically significant interaction $\left[F_{(30,6540)}=4.8771, p<0.0001\right]$ among the factors Group (AD, Nold), ROI pairs (frontal left-frontal right, central left-central right, parietal left-parietal right, occipital left-occipital right, temporal left-temporal right, limbic left-limbic right), and Band (delta, theta, alpha 1, alpha 2, beta 1, beta 2, gamma) and $p$-values of the $t$-tests for each composite EEG marker.

\begin{tabular}{|c|c|c|c|c|c|c|}
\hline \multicolumn{7}{|c|}{$p$-values of the ANOVA and $t$-test } \\
\hline & Frontal & Central & Parietal & Occipital & Temporal & Limbic \\
\hline Delta & n.s. & n.s. & n.s. & 0.0007 & n.s. & n.s. \\
\hline Theta & n.s. & n.s. & n.s. & n.s. & n.s. & n.s. \\
\hline Alpha1 & 0.00003 & 0.01 & 0.000003 & 0.00001 & 0.008 & 0.000001 \\
\hline Alpha2 & n.s. & n.s. & n.s. & 0.01 & n.s. & 0.001 \\
\hline Delta/Alpha1 & - & - & - & 0.03 & - & - \\
\hline Delta/Alpha2 & - & - & - & n.s. & - & - \\
\hline
\end{tabular}

higher delta current density in frontal, central, parietal, occipital, temporal, and limbic regions, as well as a higher theta current density in the frontal region. The $\mathrm{AD}$ group also showed a lower alpha 1 current density in frontal, central, parietal, occipital, temporal, and limbic regions, as well as a lower alpha 2 current density in central, parietal, occipital, temporal, and limbic regions.

\section{Results of the Second Statistical Session: Lagged Linear Connectivity (LLC)}

Figure 3 shows the grand average across subjects of the interhemispherical LLC values relative to a statistically significant ANOVA interaction $\left[F_{(30,6540)}=4.8771, p<0.0001\right]$ among the factors Group ( $\mathrm{AD}$, Nold), ROI pairs (frontal left-frontal right, central left-central right, parietal left-parietal right, occipital leftoccipital right, temporal left-temporal right, limbic left-limbic right), and Band (delta, theta, alpha 1, alpha 2, beta 1, beta 2, gamma). Subjects' age, education, IAF, and gender were used as covariates. Planned post-hoc testing exhibited the following results (see Table 4). Compared to the Nold group, the AD group showed higher delta inter-hemispherical LLC at occipital region, lower alpha 1 inter-hemispherical LLC at frontal, central, parietal, occipital, temporal, and limbic regions, as well as a lower alpha 2 inter-hemispherical LLC at occipital and limbic regions.

Figure 4 shows the grand average across subjects of the left intra-hemispherical LLC values relative to a statistically significant ANOVA interaction $\left[F_{(84,18312)}=8.5942, p<\right.$ 


\section{STATISTICAL ANOVA INTERACTION AMONG GROUP, BAND, ROI PAIRS}
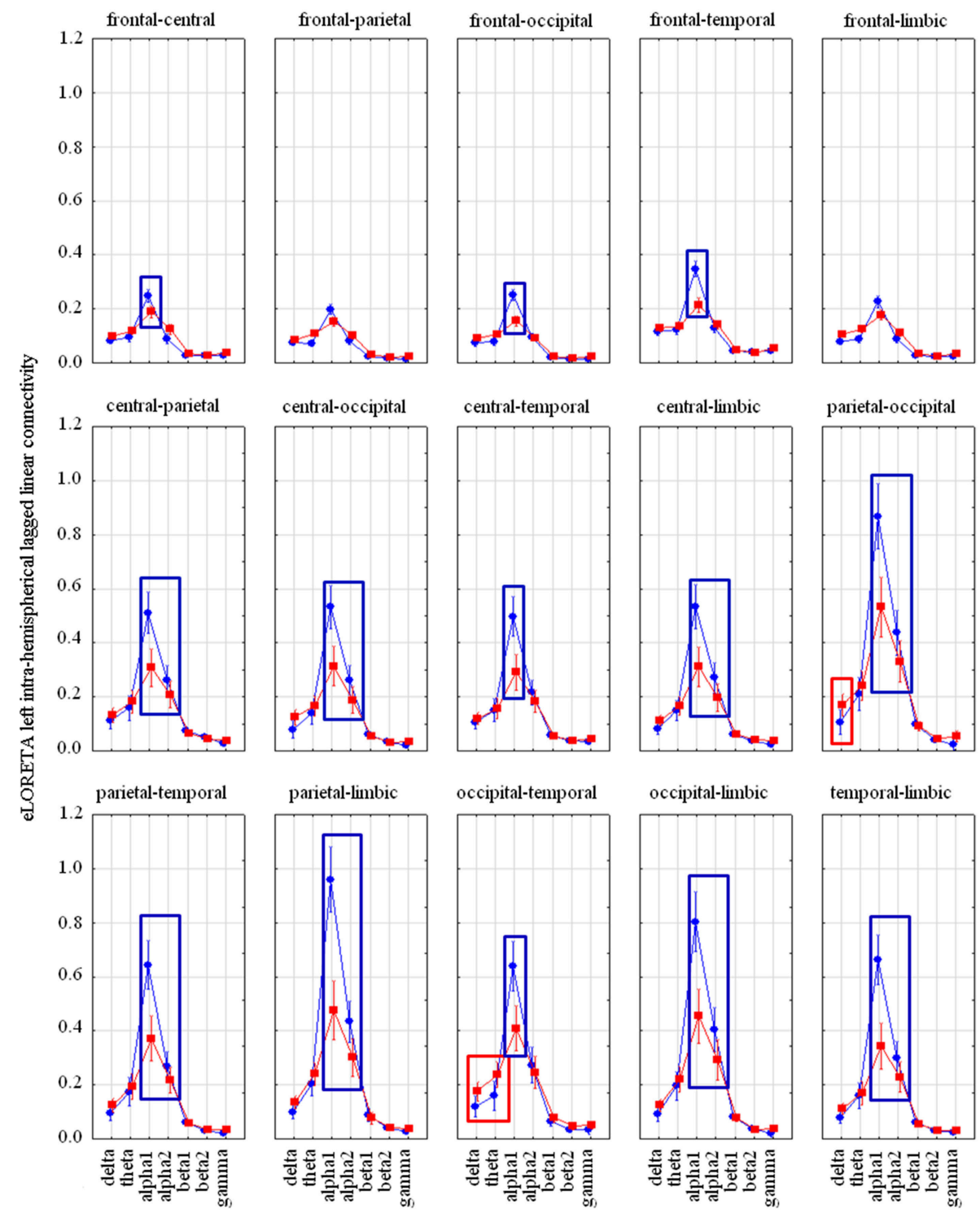

central-occipital
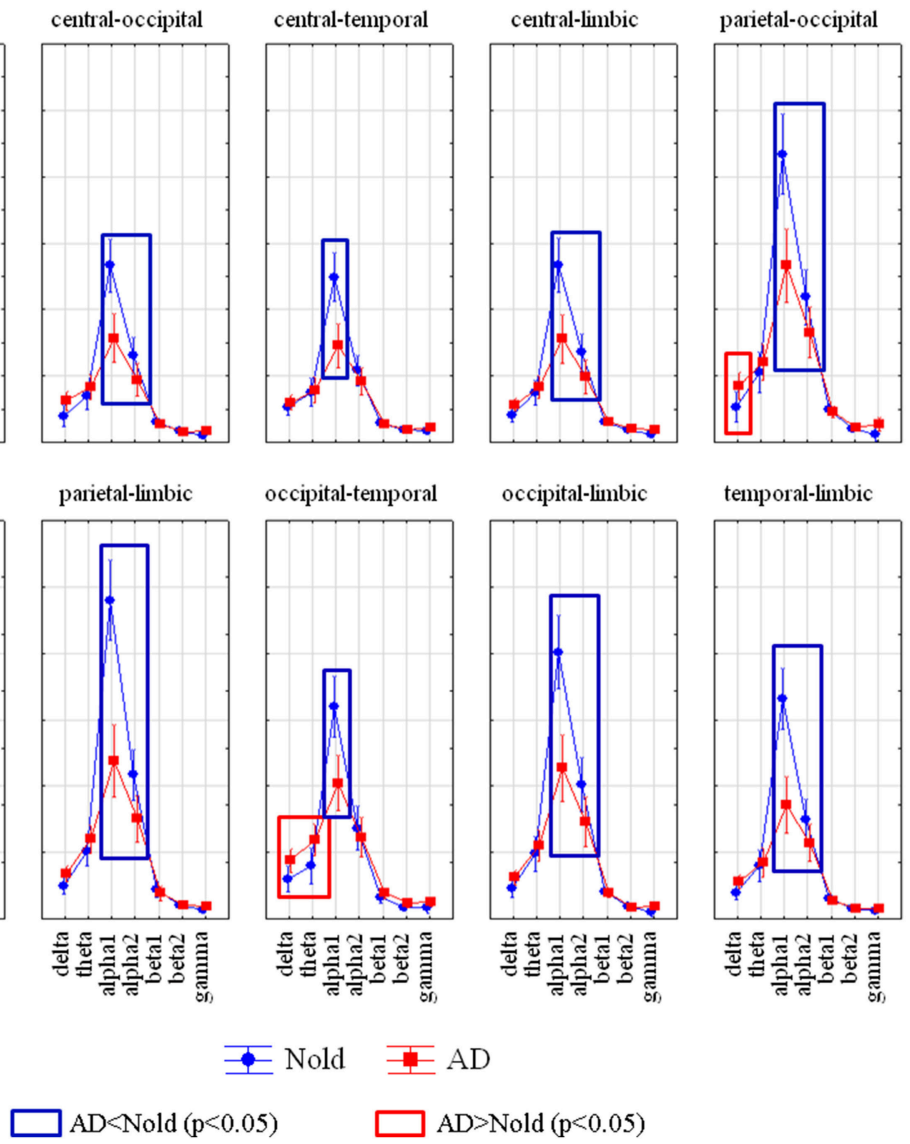

FIGURE 4 | Diagram showing the grand average of the EEG intra-hemispherical lagged linear connectivity computed between each pair of regions of interest (ROI) in the left hemisphere. These values refer a statistically significant ANOVA interaction $\left[F_{(84,18312)}=8.5942, p<0.0001\right]$ among the factors Group (AD, Nold), ROI pairs (frontal-central, frontal-parietal, frontal-occipital, frontal-temporal, frontal-limbic, central-parietal, central-occipital, central, temporal, central-limbic, parietal-occipital, parietal-temporal, parietal-limbic, occipital-temporal, occipital-limbic, and temporal-limbic), and Band (delta, theta, alpha 1, alpha 2, beta 1, beta 2, gamma). Subjects' age, education, IAF, and gender were used as covariates. Legend: the rectangles indicate the ROI pairs and frequency bands in which connectivity values presented the pattern Nold $\neq A D$ (Duncan test, $p<0.05$ ).

0.0001] among the factors Group (AD, Nold), ROI pairs (frontalcentral, frontal-parietal, frontal-occipital, frontal-temporal, frontal-limbic, central-parietal, central-occipital, centraltemporal, central-limbic, parietal-occipital, parietal-temporal, parietal-limbic, occipital-temporal, occipital-limbic, and temporal-limbic), and Band (delta, theta, alpha 1, alpha 2, beta 1, beta 2, gamma). Subjects' age, education, IAF, and gender were used as covariates. Planned post-hoc testing 
TABLE $5 \mid p$-values (Duncan post-hoc) of the ANOVA related to the comparisons of left intra-hemispherical lagged linear connectivity showing a statistically significant interaction $\left[F_{(84,18312)}=8.5942, p<0.0001\right]$ among the factors Group (AD, Nold), ROI pairs (frontal-central, frontal-parietal, frontal-occipital, frontal-temporal, frontal-limbic, central-parietal, central-occipital, central-temporal, central-limbic, parietal-occipital, parietal-temporal, parietal-limbic, occipital-temporal, occipital-limbic, and temporal-limbic), and Band (delta, theta, alpha 1, alpha 2, beta 1, beta 2, gamma) and $p$-values of the $t$-tests for each composite EEG marker.

\begin{tabular}{|c|c|c|c|c|c|}
\hline & Delta & Theta & \multicolumn{2}{|c|}{$p$-values of the ANOVA and $t$-tes } & Delta/Alpha1 \\
\hline Frontal-parietal & n.s. & n.s. & n.s. & n.s. & - \\
\hline Frontal-occipital & n.s. & n.s. & 0.00008 & n.s. & - \\
\hline Frontal-temporal & n.s. & n.s. & 0.000001 & n.s. & - \\
\hline Central-parietal & n.s. & n.s. & 0.000002 & 0.03 & - \\
\hline Central-occipital & n.s. & n.s. & 0.000002 & n.s. & - \\
\hline Central-temporal & n.s. & n.s. & 0.000002 & n.s. & - \\
\hline Central-limbic & n.s. & n.s. & 0.000002 & 0.03 & - \\
\hline Parietal-occipital & 0.01 & n.s. & 0.000005 & 0.000006 & 0.02 \\
\hline Temporal-limbic & n.s. & n.s. & 0.000002 & 0.002 & - \\
\hline
\end{tabular}

exhibited the following results (see Table 5). Compared to the Nold group, the AD group showed higher delta left intrahemispherical LLC at parietal-occipital and occipital-temporal pairs as well as higher theta left intra-hemispherical LLC at occipital-temporal pair. Besides, the $\mathrm{AD}$ group showed lower alpha 1 left intra-hemispherical LLC at frontalcentral, frontal-occipital, frontal-temporal, central-parietal, central-occipital, central-temporal, central-limbic, parietaloccipital, parietal-temporal, parietal-limbic, occipital-temporal, occipital-limbic, and temporal-limbic pairs as well as a lower alpha 2 left intra-hemispherical LLC at central-parietal, central-occipital, central-limbic, parietal-occipital, parietaltemporal, parietal-limbic, occipital-limbic, and temporal-limbic pairs.

Figure 5 shows the grand average across subjects of the right intra-hemispherical LLC values relative to a statistically significant ANOVA interaction $\left[F_{(84,18312)}=4.6296, p<\right.$ 0.0001] among the factors Group (AD, Nold), ROI pairs (frontal-central, frontal-parietal, frontal-occipital, frontaltemporal, frontal-limbic, central-parietal, central-occipital, central-temporal, central-limbic, parietal-occipital, parietaltemporal, parietal-limbic, occipital-temporal, occipital-limbic, and temporal-limbic), and Band (delta, theta, alpha 1, alpha 2, beta 1 , beta 2 , gamma). Subjects' age, education, IAF, and gender were used as covariates. Planned post-hoc testing exhibited the following results (see Table 6). Compared to the Nold group, the $\mathrm{AD}$ group showed higher theta right intra-hemispherical LLC at occipital-temporal pair. Besides, the AD group showed lower alpha 1 right intra-hemispherical LLC at all the ROI pairs, as well as a lower alpha 2 right intra-hemispherical LLC at central-occipital, central-limbic, parietal-occipital, parietal-limbic, occipital-temporal, occipital-limbic, and temporal-limbic pairs.

\section{Results of the Third Statistical Session: Accuracy of the EEG Markers}

The results of the first statistical session showed that compared to the Nold group, the AD group was characterized by higher delta source activity and lower alpha 1 source activity in several cortical regions, as well as higher theta activity in frontal region. Therefore, we decided to use composite EEG markers of source activity besides the simple EEG markers. This decision allowed an integration of the information content conveyed by the EEG markers of current density. Specifically, the composite EEG markers were obtained by computing the ratio between the source activities of the different EEG ryhthms when these activities were statistically abnormal in the same cortical region of interest. In addition, an independent $t$-test analysis were performed for each composite EEG marker (see Table 3), in order to ensure that the statistical differences continued being significant for the composite markers. Those composite EEG markers which not showed significant differences between the groups were not included as discriminant variables for the analysis of the ROC curves.

In total, the following composite EEG markers of current density were formed: (1) delta/alpha 1 current density in frontal region; (2) delta/alpha 1 current density in central region; (3) delta/alpha 1 current density in parietal region; (4) delta/alpha 1 current density in occipital region; (5) delta/alpha 1 current density in temporal region; (6) delta/alpha 1 current density in limbic region; (7) theta/alpha 


\section{STATISTICAL ANOVA INTERACTION AMONG GROUP, BAND, ROI PAIRS}
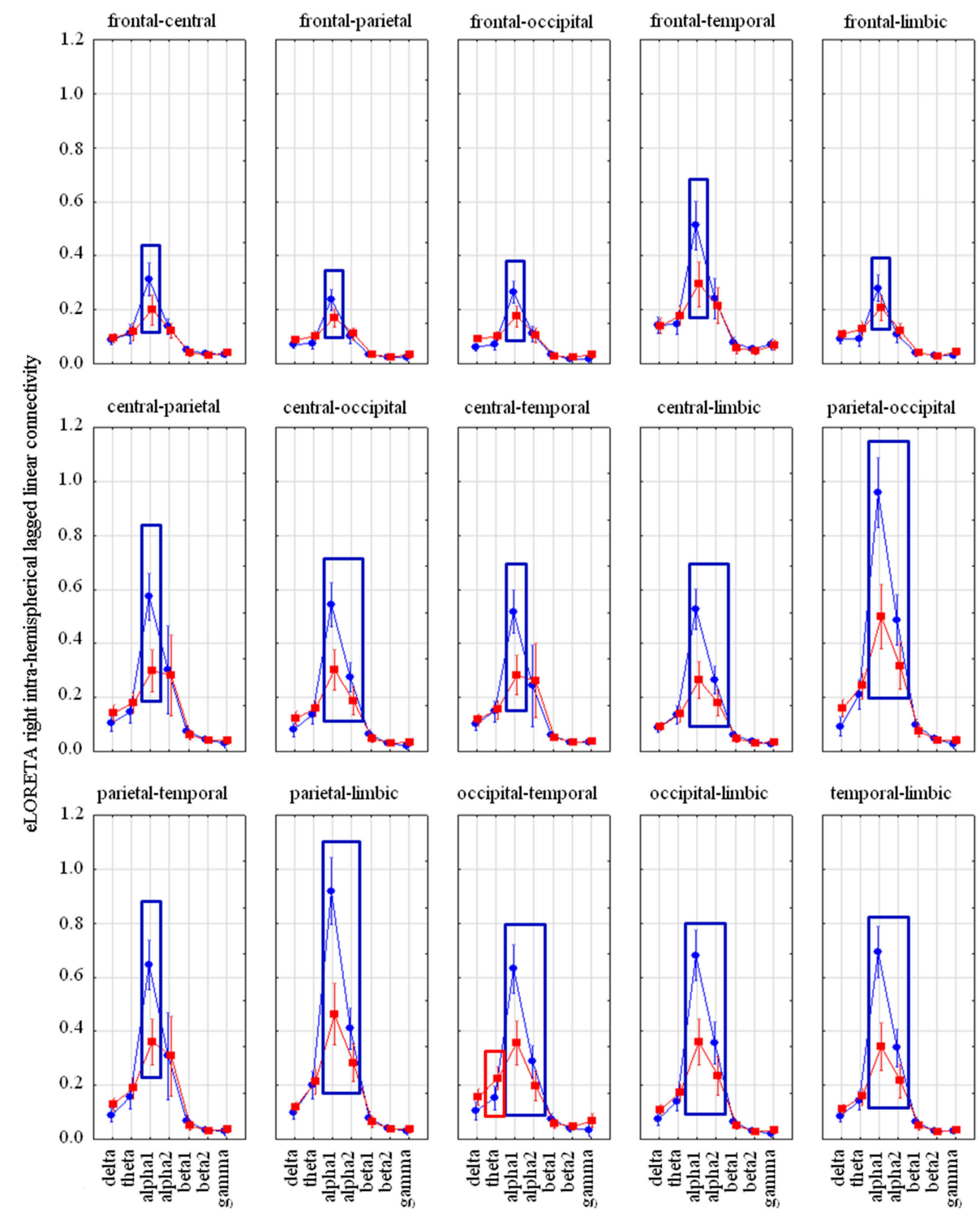

central-occipital
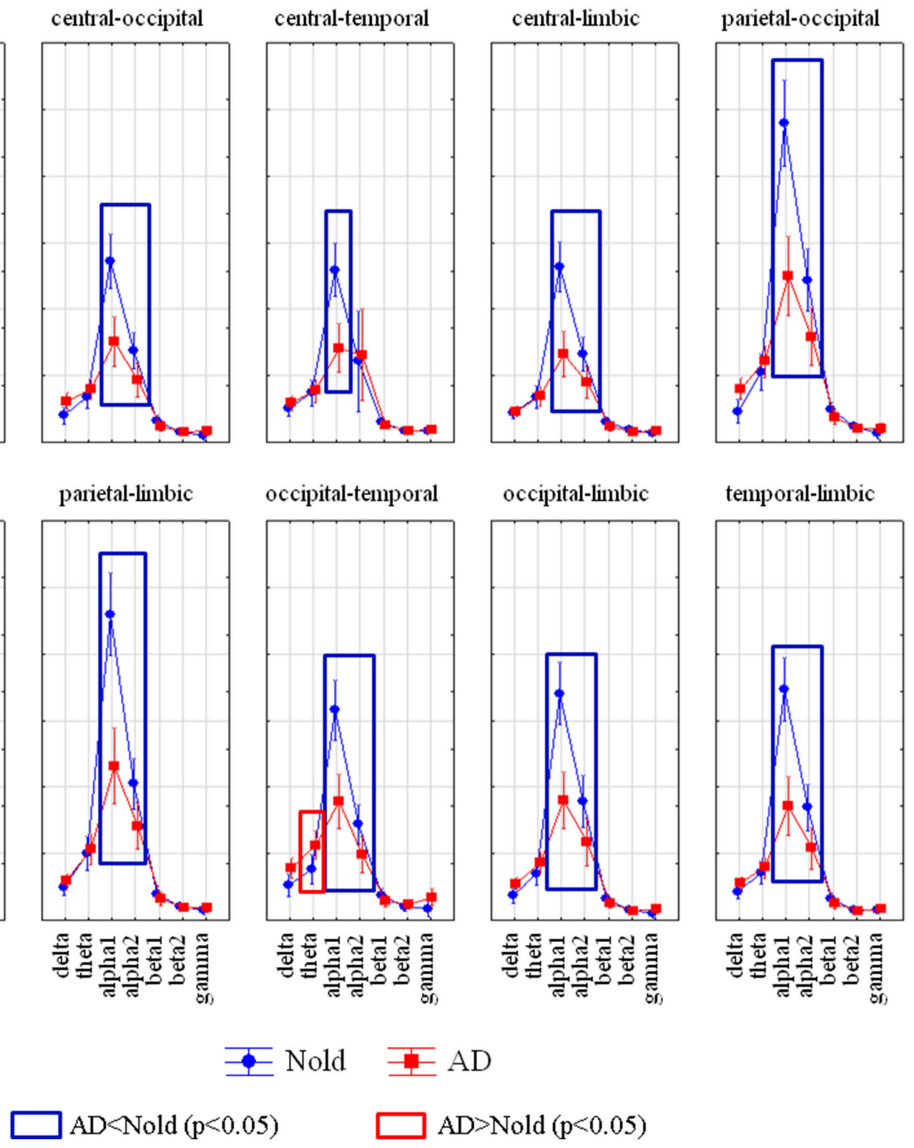

Nold

$\mathrm{AD}$

FIGURE 5 | Diagram showing the grand average of the EEG intra-hemispherical lagged linear connectivity computed between each pair of regions of interest (ROI) in the right hemisphere. These values refer a statistically significant ANOVA interaction $\left[F_{(84,18312)}=4.6296, p<0.0001\right]$ among the factors Group (AD, Nold), ROI pairs (frontal-central, frontal-parietal, frontal-occipital, frontal-temporal, frontal-limbic, central-parietal, central-occipital, central-temporal, central-limbic, parietal-occipital, parietal-temporal, parietal-limbic, occipital-temporal, occipital-limbic, and temporal-limbic), and Band (delta, theta, alpha 1, alpha 2, beta 1, beta 2, gamma). Subjects' age, education, IAF, and gender were used as covariates. Legend: the rectangles indicate the ROI pairs and frequency bands in which connectivity values presented the pattern Nold $\neq \mathrm{AD}$ (Duncan test, $p<0.05$ ).

1 current density in frontal region; (8) delta/alpha 2 current density in central region; (9) delta/alpha 2 current density in parietal region; (10) delta/alpha 2 current density in occipital region; (11) delta/alpha 2 current density in temporal region; (12) delta/alpha 2 current density in limbic region

The results of the second statistical session also showed that compared to the Nold group, the $\mathrm{AD}$ group was characterized by 
TABLE $6 \mid p$-values (Duncan post-hoc) of the ANOVA related to the comparisons of right intra-hemispherical lagged linear connectivity showing a statistically significant interaction interaction $\left[F_{(84,18312)}=4.6296, p<0.0001\right]$ among the factors Group (AD, Nold), ROI pairs (frontal-central, frontal-parietal, frontal-occipital, frontal-temporal, frontal-limbic, central-parietal, central-occipital, central-temporal, central-limbic, parietal-occipital, parietal-temporal, parietal-limbic, occipital-temporal, occipital-limbic, and temporal-limbic), and Band (delta, theta, alpha 1, alpha 2, beta 1, beta 2, gamma) and $p$-values of the $t$-tests for each composite EEG marker.

\begin{tabular}{|c|c|c|c|c|c|c|}
\hline \multicolumn{7}{|c|}{$p$-values of the ANOVA and $t$-test } \\
\hline Frontal-parietal & n.s. & n.s. & 0.04 & n.s. & - & - \\
\hline Frontal-occipital & n.s. & n.s. & 0.006 & n.s. & - & - \\
\hline Frontal-temporal & n.s. & n.s. & 0.000002 & n.s. & - & - \\
\hline Central-parietal & n.s. & n.s. & 0.000001 & n.s. & - & - \\
\hline Central-occipital & n.s. & n.s. & 0.000001 & 0.007 & - & - \\
\hline Central-temporal & n.s. & n.s. & 0.000001 & n.s. & - & - \\
\hline Central-limbic & n.s. & n.s. & 0.000001 & 0.03 & - & - \\
\hline Parietal-occipital & n.s. & n.s. & 0.000002 & 0.000001 & - & - \\
\hline Temporal-limbic & n.s. & n.s. & 0.000002 & 0.0007 & - & - \\
\hline
\end{tabular}

abnormal inter- and intra-hemispherical LLC in several cortical regions. Therefore, the same procedure was followed to form composite EEG markers of connectivity (see $p$-values for the $t$ tests in Tables 4-6). The following composite EEG markers of LLC were formed: (1) delta/alpha 1 inter-hemispherical LLC in occipital region; (2) delta/alpha 1 left intra-hemispherical LLC in parietal-occipital pair; (3) delta/alpha 1 left intra-hemispherical LLC in occipital-temporal pair; (4) theta/alpha 1 left intrahemispherical LLC in occipital-temporal pair; (5) theta/alpha 1 right intra-hemispherical LLC in occipital-temporal pair; (6) theta/alpha 2 right intra-hemispherical LLC in occipital-temporal pair.

The following 15 EEG markers overcome the threshold of $70 \%$ stablished as "moderate" classification rate (see Table 7): (1) Delta/alpha 1 current density in central region; (2) Delta/alpha 1 current density in parietal region; (3) Delta/alpha 1 current density in occipital region; (4) Delta/alpha 1 current density in temporal region; (5) Delta/alpha 1 current density in limbic region; (6) Theta/alpha 1 current density in frontal region; (7) Delta/alpha 2 current density in central region; (8) Delta/alpha 2 current density in parietal region; (9) Delta/alpha 2 current density in occipital region; (10) Delta/alpha 2 current density in temporal region; (11) Delta/alpha 2 current density in limbic region; (12) Delta/alpha 1 inter-hemispherical LLC in occipital region; (13) Theta/alpha 1 left intra-hemispherical LLC in occipital-temporal pair; (14) Alpha 1 right intrahemispherical LLC in parietal-limbic pair; (15) Theta/alpha 1 right intra-hemispherical LLC in occipital-temporal pair.

Among these EEG markers, delta/alpha 1 current density in occipital region reached the following best classification rate: the specificity of $78 \%$, the sensitivity of $73.3 \%$, the accuracy of $75.5 \%$, and AUROC of $82 \%$. Regarding the EEG markers of LLC, theta/alpha 1 right intra-hemispherical LLC in occipital-temporal pair reached the best classification rate: the specificity of $72 \%$, the sensitivity of $70.8 \%$, the accuracy of $71.4 \%$, and AUROC of $74 \%$. Figure 6 shows the ROC curves for these both composite EEG markers.

\section{Results of the Fourth Statistical Session: Relationship between the EEG Markers and Relevant Variables of AD}

The MMSE score as an index of global cognition was on average $19.9( \pm 0.5 \mathrm{SD})$ in the AD-subgroup $(n=53)$ and $18.2( \pm 0.4 \mathrm{SD})$ in the $\mathrm{AD}+$ subgroup $(n=67)$. Independent $t$-test indicated that the difference of the MMSE score between the two AD subgroups was statistically significant $(p<0.001)$, in line with the working hypothesis.

Table 8 reports information about personal and clinical characteristics of the $\mathrm{AD}-$ and $\mathrm{AD}+$ individuals of 39 $\mathrm{AD}$ patients having MRI associated with EEG recordings. Concerning the MRI markers of structural brain integrity, the $\mathrm{GM}, \mathrm{WM}$, and CSF normalized volumes of the AD- subgroup $(n=12)$ were on average 0.55 ( \pm 0.003 Standard Error-SE), 0.44 $( \pm 0.009 \mathrm{SE})$, and $0.35( \pm 0.005 \mathrm{SE})$, respectively. Furthermore, they were $0.54( \pm 0.004 \mathrm{SE}), 0.43( \pm 0.006 \mathrm{SE})$, and $0.37( \pm 0.005$ $\mathrm{SE})$, respectively, in the $\mathrm{AD}+$ subgroup $(N=27)$. The ANOVA design showed a statistically significant interaction $\left[F_{(2,74)}=\right.$ 3.3761, $p<0.05$ ] between the factors Group (AD- subgroup, $\mathrm{AD}+$ subgroup; independent variable) and Volume (GM, WM, $\mathrm{CSF}$ ). Duncan post-hoc testing indicated that the GM normalized 
TABLE 7 | Results of the classification between single AD and Nold subjects based on EEG markers of source activity and lagged linear connectivity.

\begin{tabular}{|c|c|c|c|c|}
\hline LORETA sources & Sensitivity (\%) & Specificity (\%) & Accuracy (\%) & AUROC \\
\hline Central delta/alpha 1 & 71.7 & 71 & 71.4 & 0.73 \\
\hline Parietal delta/alpha 1 & 74.2 & 73 & 73.6 & 0.79 \\
\hline Occipital delta/alpha 1 & 73.3 & 78 & 75.5 & 0.82 \\
\hline Temporal delta/alpha 1 & 78.3 & 70 & 74.5 & 0.78 \\
\hline Limbic delta/alpha 1 & 75.8 & 72 & 74. & 0.77 \\
\hline Parietal delta/alpha 2 & 68.3 & 76 & 71.8 & 0.77 \\
\hline Occipital delta/alpha 2 & 71.7 & 75 & 73.2 & 0.79 \\
\hline Temporal delta/alpha 2 & 63.3 & 78 & 70 & 0.76 \\
\hline Limbic delta/alpha 2 & 66.7 & 74 & 70 & 0.73 \\
\hline Occipital delta/alpha 2 inter-hemispherical LLC & 65.8 & 66 & 65.9 & 0.70 \\
\hline
\end{tabular}

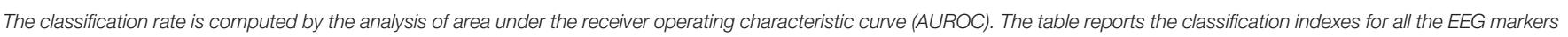
having an AUROC higher than 0.70 (i.e., $70 \%)$.

volume was lower in the $\mathrm{AD}+$ subgroup compared to the $\mathrm{AD}-$ subgroup ( $p<0.05$ ), while the CSF normalized volume was higher in the $\mathrm{AD}+$ subgroup compared to the $\mathrm{AD}$ - subgroup ( $p<0.001$; see Figure 7). Instead, the between-groups difference in the WM volume did not reach the statistical significance $(p>0.05)$.

\section{DISCUSSION}

Our "BRAINON" research program has been testing markers of resting state eyes-closed EEG rhythms to characterize neurophysiological correlates of $\mathrm{AD}$ at group level (Babiloni et al., 2004a,b, 2008, 2009a, 2010, 2011a,b,c, 2013a,b,c). These markers were extracted by LORETA/eLORETA, popular software aimed at estimating power and connectivity of cortical sources of EEG rhythms (Pascual-Marqui et al., 1994, 2007a). Results at group level showed abnormalities of these EEG markers especially at delta and alpha frequency bands (Babiloni et al., 2004a,b, 2008, 2009a, 2010, 2011a,b,c, 2013a,b,c). In the present study, we moved from group to the individual level. We tested the ability of some of these spectral EEG markers to classify single Nold subjects and AD patients with dementia, as a preliminary step toward their use for a neurophysiological assessment of AD patients.

A preliminary control analysis of the present study confirmed previous evidence of our research group (Babiloni et al., 2004a,b, 2008, 2009a, 2010, 2011a,b,c, 2013a,b,c). Concerning the cortical sources of the EEG power, main results showed a higher activity of the posterior delta sources together with lower activity of the alpha sources in the AD group compared to the Nold group. To integrate this information content, we produced composite EEG markers by computing the ratio between the activity of posterior delta and alpha sources in relevant cortical regions.
Concerning the functional connectivity of the EEG sources, the present results displayed a lower intra- and inter-hemispherical connectivity at alpha rhythms in the $\mathrm{AD}$ group compared to the Nold group. Of note, the use of eLORETA allowed overcoming the methodological limitations due to the use of standard FFT to compute the bivariate spectral coherence between electrode pairs. With respect to multivariate estimators like eLORETA, bivariate measures of EEG functional interrelatedness may produce spurious connections due to the common feeding effect (i.e., false coupling between sources $\mathrm{B}$ and $\mathrm{C}$ due to the common influence from a source A) and head volume conduction effects (Blinowska and Kaminski, 2013; Kaminski and Blinowska, 2014).

As a novelty, the present ROC curve analysis indicated that some EEG markers of intra- and inter-hemispherical source connectivity at low-frequency alpha had an AUROC higher than $70 \%$, which is the threshold of a moderate classification rate (DeLong et al., 1988). Among them, the best classification rate was reached by the functional connectivity between right occipital and temporal sources in the ratio between low-frequency alpha and theta: the sensitivity of $70.8 \%$, the specificity of $72 \%$, the accuracy of $71.4 \%$, and AUROC of $74 \%$.

In the same line, the ROC curve analysis showed that some EEG markers of cortical sources of EEG power had an AUROC higher than 70\%. They mainly comprised the ratio between lowfrequency alpha and delta source activity in posterior cortical regions. Among them, the best classification rate was reached by the ratio between occipital low-frequency alpha and delta source activity: the sensitivity of $73.3 \%$, the specificity of $78 \%$, the accuracy of $75.5 \%$, and AUROC of $82 \%$.

Overall, these present results pointed to a moderate classification rate of EEG markers of source power and 


\section{ROC CURVES}
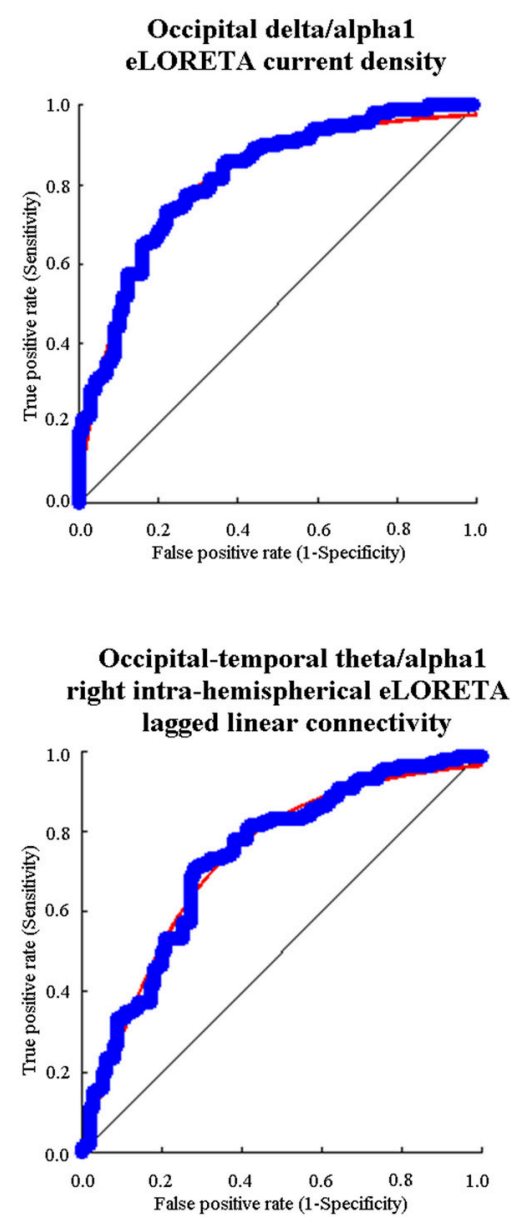

FIGURE 6 | Diagram showing the ROC (receiver operating characteristic) curves that illustrate the performance of the EEG markers with the best classification rate of single Nold and AD individuals. The upper panel shows the best EEG marker for source activity and the bottom panel the best EEG maker for functional connectivity. These EEG markers had an area under the ROC curve (AUROC) higher than 0.70 (i.e., $70 \%$ ), which is the threshold of a moderate classification performance.

functional connectivity for the detection of single AD individuals. In precedence, several studies tested various spectral EEG markers for the classification of the Nold and AD individuals. They reported classification rates between 82 and $90 \%$ using advanced mathematical classifiers including the ANNs (Anderer et al., 1994; Pritchard et al., 1994; Adler et al., 2003; Brassen et al., 2004; Lehmann et al., 2007; Dauwels et al., 2009).

\section{Clinical Significance of the EEG Topographic Markers of AD}

What is the potential clinical significance of the present EEG markers? According to the new guidelines of 2014 (Dubois et al., 2014), these EEG markers should not be used to formulate
TABLE 8 | Demographic and clinical data of the subgroups of AD patients (AD- and $A D+$ ) in the subpopulation of 39 AD patients having MRI data associated to EEG recordings.

\begin{tabular}{lcccr}
\hline & $\begin{array}{c}\text { Gender } \\
\text { (Female/Male) }\end{array}$ & $\begin{array}{c}\text { Age } \\
\text { (years) }\end{array}$ & $\begin{array}{c}\text { Education } \\
\text { (years) }\end{array}$ & \multicolumn{1}{c}{$\begin{array}{c}\text { MMSE } \\
\text { (score) }\end{array}$} \\
\hline $\mathrm{AD}-(n=12)$ & $10 / 2$ & $73.4 \pm 1.7 \mathrm{SE}$ & $7.8 \pm 1.7 \mathrm{SE}$ & $19.8 \pm 1.3 \mathrm{SE}$ \\
$\mathrm{AD}+(n=27)$ & $16 / 11$ & $66.9 \pm 1.5 \mathrm{SE}$ & $8.5 \pm 1.0 \mathrm{SE}$ & $17 \pm 0.6 \mathrm{SE}$ \\
\hline
\end{tabular}

MMSE, Mini Mental State Evaluation.

a diagnosis of $\mathrm{AD}$. Indeed, they do not directly reflect the pathophysiological markers of $\mathrm{AD}$ such as $\mathrm{A} \beta 1-42$ and tau in the cerebrospinal fluid or brain. Rather, they should be considered as candidate EEG topographic markers for the assessment of the neurophysiological mechanisms underlying the fluctuation of cortical arousal and vigilance in the relaxed wakefulness. Indeed, these neurophysiological mechanisms generate cortical delta and alpha rhythms in healthy humans, which derange in $\mathrm{AD}$ patients (Babiloni et al., 2015).

The present results suggest that EEG markers of source power and functional connectivity in relaxed wakefulness may enrich the neurophysiological assessment of $\mathrm{AD}$ patients with dementia, although the best EEG marker for the classification between Nold and $\mathrm{AD}$ individuals was the occipital sources of delta/low frequency alpha. This best EEG marker may be useful to differentiate the status of brain function in AD patients with similar values in MMSE, autonomy in the daily life, and cognitive reserve as indexed by education years and intellectual occupations across lifespan (Snowdon, 2003; Riley et al., 2005; Tyas et al., 2007). Cognition, daily life abilities, and cognitive reserve status being equal, an $\mathrm{AD}$ patient with an abnormality of the EEG marker (e.g., one standard deviation beyond the mean value of a reference Nold population) may reflect more neurophysiological "frailty" and less brain reserve than an $\mathrm{AD}$ patient negative for this EEG marker. In this example, the $\mathrm{AD}$ patient with neurophysiological "frailty" should receive more therapeutic resources and clinical attention. This clinical perspective is supported by the present evidence showing that compared to the $\mathrm{AD}$ patients with normal EEG marker, those with an abnormal EEG marker (e.g., one standard deviation beyond the mean value of a reference Nold population) did exhibit lower MMSE score, lower normalized cortical gray matter density, and greater normalized cerebrospinal fluid, as signs of an impairment of global cognition and brain structural integrity, respectively.

\section{Methodological Remarks}

Compared to the mentioned classification rates of the previous studies, the moderate classification rate of this study was reasonably due to some intrinsic methodological limitations. First, our large clinical and EEG dataset (i.e., $120 \mathrm{AD}$ vs. 100 Nold) was acquired by several clinical units, thus increasing the variance of the data due to a possible slightly different local implementation of clinical exclusion/inclusion criteria and EEG procedures. Second, no AD patient with severe dementia was enrolled in the present study (they typically show highly 


\section{NORMALIZED BRAIN VOLUMES}

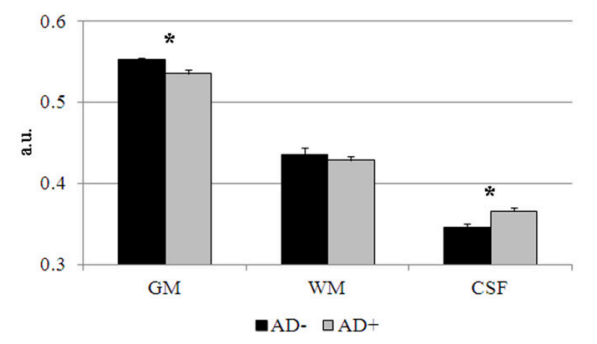

FIGURE 7 | Mean and SD-values of cortical gray matter (GM), subcortical white matter (WM), and cerebrospinal fluid (CSF) normalized volumes as indexes of brain structural integrity extracted by magnetic resonance imaging (MRI) in a subpopulation of 39 AD patients having MRI data associated to EEG recordings. The values are reported in the AD subgroup negative to the EEG marker (AD-; $n=12$ ) and in the $A D$ subgroup positive to the $E E G$ marker ( $A D+; n=27)$. These values refer to an ANOVA design showing a statistically significant interaction $\left[F_{(2,74)}=\right.$ 3.3761, $p<0.05$ ] between the factors Group (AD-, AD+) and Volume (GM, WM, CSF). Asterisks indicate the $p$ level of the statistical differences between the two AD subgroups obtained by Duncan post-hoc testing.

abnormal delta and alpha rhythms, which inflate classification rate). Third, all enrolled $\mathrm{AD}$ patients were under treatment by standard long-term Acetylcholinesterase inhibitors, which are expected to preserve alpha rhythms and reduce the difference between Nold and AD subjects in this respect (Brassen and Adler, 2003; Geldmacher, 2003; Onofrj et al., 2003; Babiloni et al., 2006d). Fourth, some of the enrolled Nold subjects (chosen among $\mathrm{AD}$ relatives or caregivers) might indeed suffer from a preclinical stage of $\mathrm{AD}$, thus confounding the classification task. In fact, they were diagnosed based on traditional clinical criteria (e.g., NINCDS-ADRDA, McKhann et al., 1984; DSM-IV), without an assessment of the pathophysiological markers of AD (Dubois et al., 2014). Of note, we mitigated this risk including only Nold subjects having 27 or higher score of MMSE.

\section{CONCLUSIONS}

In our previous studies, spectral EEG markers of relaxed wakefulness were found to be abnormal in groups of $\mathrm{AD}$ patients with dementia (Babiloni et al., 2004a, 2006a, 2009b, 2011a,b,

\section{REFERENCES}

Adler, G., Brassen, S., and Jajcevic, A. (2003). EEG coherence in Alzheimer's dementia. J. Neural Transm. 110, 1051-1058. doi: 10.1007/s00702-003-0024-8

Anderer, P., Saletu, B., Klöppel, B., Semlitsch, H. V., and Werner, H. (1994). Discrimination between demented patients and normals based on topographic EEG slow wave activity: comparison between $\mathrm{z}$ statistics, discriminant analysis and artificial neural network classifiers. Electroencephalogr. Clin. Neurophysiol. 91, 108-117. doi: 10.1016/0013-4694(94) 90032-9

Ashburner, J., and Friston, K. (1997). Multimodal image coregistration and partitioning-A unified framework. Neuroimage 6, 209-217. doi: 10.1006/nimg.1997.0290

Babiloni, C., Binetti, G., Cassetta, E., Cerboneschi, D., Dal Forno, G., Del Percio, C., et al. (2004a). Mapping distributed sources of cortical rhythms in mild 2013b). Can these EEG markers be used for the classification of single Nold and $\mathrm{AD}$ individuals, as a premise for future clinical applications? The results of the present study showed that 15 spectral EEG markers of source power and functional connectivity had a classification rate higher than $70 \%$, computed by the analysis of ROC curves. Most of them were based on the ratio between alpha and delta source power in posterior cortical regions. The best classification rate was reached by the ratio between occipital low-frequency alpha and delta source activity. It exhibited the following performance: the sensitivity of $73.3 \%$, the specificity of $78 \%$, the accuracy of $75.5 \%$, and AUROC of $82 \%$. These results pointed to a moderate classification rate of several EEG markers of source power and functional connectivity for the detection of single AD individuals. Future studies will have to test the classification rate of these relevant EEG markers as a multivariate input to advanced mathematical classifiers such as the ANNs.

\section{AUTHOR CONTRIBUTIONS}

$\mathrm{CB}, \mathrm{RF}, \mathrm{AT}, \mathrm{RL}, \mathrm{CP}$, and $\mathrm{VB}$ gave their contributions to the conception and design of the work. AS, RF, FN, LG, RT, VC, $\mathrm{MB}, \mathrm{AG}$, PS, SA, GB, GS, GL, and GF were responsible for the enrollments, all the clinical evaluations, and the recordings of EEG data. AT, RL, SC, JM, AB, and CP performed the analysis of data. CP, RL, GT, and VB performed the statistical analysis. CB, $\mathrm{AT}, \mathrm{RL}$, and $\mathrm{CP}$ gave their contribution in the interpretation of the results. $\mathrm{CB}, \mathrm{AT}, \mathrm{RL}$, and $\mathrm{CP}$ contributed in drafting the work and revising it critically for important intellectual content. AS, RF, FN, LG, GL, GF, and AB contributed in the revision of the first and later versions of the manuscript.

\section{ACKNOWLEDGMENTS}

The present study was developed and granted in the framework of the following projects: "Smart Health 2.0" (Italian Ministry of University and Technological Research, Pon04a2_C-MIUR D.D. 626/Ric e 703/Ric) and "CONNAGE" (Italian Ministry of University and Technological Research, PRIN2010-2011, prot. 2010SH7H3F).
Alzheimer's disease. A multicentric EEG study. Neuroimage 22, 57-67. doi: 10.1016/j.neuroimage.2003.09.028

Babiloni, C., Binetti, G., Cassetta, E., Del Forno, G., Percio, C., Del Ferreri, F., et al. (2006a). Sources of cortical rhythms change as a function of cognitive impairment in pathological aging: a multicenter study. Clin. Neurophysiol. 117, 252-268. doi: 10.1016/j.clinph.2005.09.019

Babiloni, C., Brancucci, A., Capotosto, P., Romani, G. L., ArendtNielsen, L., Chen, A. C. N., et al. (2005). Slow cortical potential shifts preceding sensorimotor interactions. Brain Res. Bull. 65, 309-316. doi: 10.1016/j.brainresbull.2004.11.023

Babiloni, C., Brancucci, A., Vecchio, F., Arendt-Nielsen, L., Chen, A. C. N., and Rossini, P. M. (2006b). Anticipation of somatosensory and motor events increases centro-parietal functional coupling: an EEG coherence study. Clin. Neurophysiol. 117, 1000-1008. doi: 10.1016/j.clinph.2005. 12.028 
Babiloni, C., Cassetta, E., Dal Forno, G., Del Percio, C., Ferreri, F., Ferri, R., et al. (2006d). Donepezil effects on sources of cortical rhythms in mild Alzheimer's disease: responders vs. Non-Responders. Neuroimage 31, 1650-1665. doi: 10.1016/j.neuroimage.2006.02.015

Babiloni, C., Del Percio, C., Bordet, R., Bourriez, J. L., Bentivoglio, M., Payoux, P., et al. (2013c). Effects of acetylcholinesterase inhibitors and memantine on resting-state electroencephalographic rhythms in Alzheimer's disease patients. Clin. Neurophysiol. 124, 837-850. doi: 10.1016/j.clinph.2012. 09.017

Babiloni, C., De Pandis, M. F., Vecchio, F., Buffo, P., Sorpresi, F., Frisoni, G. B., et al. (2011c). Cortical sources of resting state electroencephalographic rhythms in Parkinson's disease related dementia and Alzheimer's disease. Clin. Neurophysiol. 122, 2355-2364. doi: 10.1016/j.clinph.2011. 03.029

Babiloni, C., Ferri, R., Binetti, G., Vecchio, F., Frisoni, G. B., Lanuzza, B., et al. (2009a). Directionality of EEG synchronization in Alzheimer's disease subjects. Neurobiol. Aging 30, 93-102. doi: 10.1016/j.neurobiolaging.2007. 05.007

Babiloni, C., Ferri, R., Moretti, D. V., Strambi, A., Binetti, G., Dal Forno, G., et al. (2004b). Abnormal frontoparietal coupling of brain rhythms in mild Alzheimer's disease: a multicentric EEG study. Eur. J. Neurosci. 19, 2583-2590. doi: 10.1111/j.0953-816X.2004.03333.x

Babiloni, C., Frisoni, G. B., Pievani, M., Vecchio, F., Infarinato, F., Geroldi, C., et al. (2008). White matter vascular lesions are related to parietal-to-frontal coupling of EEG rhythms in mild cognitive impairment. Hum. Brain Mapp. 29, 1355-1367. doi: 10.1002/hbm.20467

Babiloni, C., Frisoni, G. B., Pievani, M., Vecchio, F., Lizio, R., Buttiglione, M., et al. (2009b). Hippocampal volume and cortical sources of EEG alpha rhythms in mild cognitive impairment and Alzheimer disease. Neuroimage 44, 123-135. doi: 10.1016/j.neuroimage.2008.08.005

Babiloni, C., Frisoni, G. B., Vecchio, F., Lizio, R., Pievani, M., Cristina, G., et al. (2011a). Stability of clinical condition in mild cognitive impairment is related to cortical sources of alpha rhythms: an electroencephalographic study. Hum. Brain Mapp. 32, 1916-1931. doi: 10.1002/hbm. 21157

Babiloni, C., Frisoni, G. B., Vecchio, F., Pievani, M., Geroldi, C., De Carli, C., et al. (2010). Global functional coupling of resting EEG rhythms is related to white-matter lesions along the cholinergic tracts in subjects with amnesic mild cognitive impairment. J. Alzheimers. Dis. 19, 859-871. doi: 10.3233/JAD-20101290

Babiloni, C., Infarinato, F., Aujard, F., Bastlund, J. F., Bentivoglio, M., Bertini, G., et al. (2013a). Effects of pharmacological agents, sleep deprivation, Hypoxia and transcranial magnetic stimulation on electroencephalographic rhythms in rodents: towards translational challenge models for drug discovery in Alzheimer's disease. Clin. Neurophysiol. 124, 437-451. doi: 10.1016/j.clinph.2012.07.023

Babiloni, C., Infarinato, F., Triggiani, A. I., Lizio, R., Del Percio, C., Marzano, N., et al. (2013b). Resting state EEG rhythms as network disease markers for drug discovery in Alzheimer's disease. Drug Discov. Today Ther. Strateg. 10, e85-e90. doi: 10.1016/j.ddstr.2014.02.003

Babiloni, C., Lizio, R., Carducci, F., Vecchio, F., Redolfi, A., Marino, S., et al. (2011b). Resting state cortical electroencephalographic rhythms and white matter vascular lesions in subjects with Alzheimer's disease: an Italian multicenter study. J. Alzheimers. Dis. 26, 331-346. doi: 10.3233/JAD-2011101710

Babiloni, C., Lizio, R., Marzano, N., Capotosto, P., Soricelli, A., Triggiani, A. I., et al. (2015). Brain neural synchronization and functional coupling in Alzheimer's disease as revealed by resting state EEG rhythms. Int. J. Psychophysiol. doi: 10.1016/j.ijpsycho.2015.02.008. [Epub ahead of print].

Besthorn, C., Zerfass, R., Geiger-Kabisch, C., Sattel, H., Daniel, S., Schreiter-Gasser, U., et al. (1997). Discrimination of Alzheimer's disease and normal aging by EEG data. Electroencephalogr. Clin. Neurophysiol. 103, 241-248.

Blinowska, K. J., and Kaminski, M. (2013). Functional brain networks: random, "small world" or deterministic? PLOS ONE 8:e78763. doi: 10.1371/journal.pone.0078763

Braak, H., and Braak, E. (1995). Staging of Alzheimer's disease-related neurofibrillary changes. Neurobiol. Aging. 16, 271-278. doi: 10.1016/0197-4580 (95)00021-6
Brassen, S., and Adler, G. (2003). Short-term effects of acetylcholinesterase inhibitor treatment on EEG and memory performance in alzheimer patients: an open, controlled trial. Pharmacopsychiatry 36, 304-308. doi: 10.1055/s-200345118

Brassen, S., Braus, D. F., Weber-Fahr, W., Tost, H., Moritz, S., and Adler, G. (2004). Late-onset depression with mild cognitive deficits: electrophysiological evidences for a preclinical dementia syndrome. Dement. Geriatr. Cogn. Disord. 18, 271-277. doi: 10.1159/000080028

Brett, M., Johnsrude, I. S., and Owen, A. M. (2002). The problem of functional localization in the human brain. Nat. Rev. Neurosci. 3, 243-249. doi: $10.1038 / \mathrm{nrn} 756$

Buscema, M., Rossini, P., Babiloni, C., and Grossi, E. (2007). The IFAST model, a novel parallel nonlinear EEG analysis technique, distinguishes mild cognitive impairment and Alzheimer's disease patients with high degree of accuracy. Artif. Intell. Med. 40, 127-141. doi: 10.1016/j.artmed.2007. 02.006

Canuet, L., Ishii, R., Pascual-Marqui, R. D., Iwase, M., Kurimoto, R., Aoki, Y., et al. (2011). Resting-state EEG source localization and functional connectivity in schizophrenia-like psychosis of epilepsy. PLoS ONE 6:e27863. doi: 10.1371/journal.pone.0027863

Chiaramonti, R., Muscas, G. C., Paganini, M., Müller, T. J., Fallgatter, A. J., Versari, A., et al. (1997). Correlations of topographical EEG features with clinical severity in mild and moderate dementia of Alzheimer type. Neuropsychobiology 36, 153-158.

Dauwels, J., Vialatte, F., Latchoumane, C., Jeong, J., and Cichocki, A. (2009). EEG synchrony analysis for early diagnosis of Alzheimer's disease: a study with several synchrony measures and EEG data sets. Conf. Proc. IEEE Eng. Med. Biol. Soc. 2009, 2224-2227. doi: 10.1109/IEMBS.2009.5334862

Dauwels, J., Vialatte, F., Musha, T., and Cichocki, A. (2010). A comparative study of synchrony measures for the early diagnosis of Alzheimer's disease based on EEG. Neuroimage 49, 668-693. doi: 10.1016/j.neuroimage.2009. 06.056

DeLong, E. R., DeLong, D. M., and Clarke-Pearson, D. L. (1988). Comparing the areas under two or more correlated receiver operating characteristic curves: a nonparametric approach. Biometrics 44, 837-845. doi: 10.2307/ 2531595

Dierks, T., Ihl, R., Frölich, L., and Maurer, K. (1993). Dementia of the Alzheimer type: effects on the spontaneous EEG described by dipole sources. Psychiatry Res. 50, 151-162. doi: 10.1016/0925-4927(93)90027-F

Dierks, T., Jelic, V., Pascual-Marqui, R. D., Wahlund, L. O., Julin, P., Linden, D. E. J., et al. (2000). Spatial pattern of cerebral glucose metabolism (PET) correlates with localization of intracerebral EEG-generators in Alzheimer's disease. Clin. Neurophysiol. 111, 1817-1824. doi: 10.1016/S1388-2457(00) 00427-2

Dubois, B., Feldman, H. H., Jacova, C., Hampel, H., Molinuevo, J. L., Blennow, K., et al. (2014). Advancing research diagnostic criteria for Alzheimer's disease: the IWG-2 criteria. Lancet Neurol. 13, 614-629. doi: 10.1016/S14744422(14)70090-0

Elmstáhl, S., and Rosén, I. (1997). Postural hypotension and EEG variables predict cognitive decline: results from a 5-year follow-up of healthy elderly women. Dement. Geriatr. Cogn. Disord. 8, 180-187. doi: 10.1159/ 000106629

Fernández, A., Turrero, A., Zuluaga, P., Gil-Gregorio, P., del Pozo, F., Maestu, F., et al. (2013). MEG delta mapping along the healthy aging-Alzheimer's disease continuum: diagnostic implications. J. Alzheimers Dis. 35, 495-507. doi: 10.3233/JAD-121912

Folstein, M. F., Folstein, S. E., and McHugh, P. R. (1975). “Mini-mental state”. A practical method for grading the cognitive state of patients for the clinician. J. Psychiatr. Res. 12, 189-198.

Förstl, H., and Kurz, A. (1999). Clinical features of Alzheimer's disease. Europ. Arch. Psychiatry Clin. Neurosci. 249, 288-290.

Fuchs, M., Kastner, J., Wagner, M., Hawes, S., and Ebersole, J. S. (2002). A standardized boundary element method volume conductor model. Clin. Neurophysiol. 113, 702-712. doi: 10.1016/S1388-2457(02) 00030-5

Geldmacher, D. S. (2003). Long-term cholinesterase inhibitor therapy for Alzheimer's disease. Prim. Care Companion J. Clin. Psychiatry 5, 251-259. doi: 10.4088/PCC.v05n0602 
Glodzik-Sobanska, L., Rusinek, H., Mosconi, L., Li, Y., Zhan, J., de Santi, S., et al. (2005). The role of quantitative structural imaging in the early diagnosis of Alzheimer's diseaseQ4. Neuroimaging Clin. N. Am. 15, 803-826. doi: 10.1016/j. nic.2005.09.004

Gómez, C., Mediavilla, A., Hornero, R., Abásolo, D., and Fernández, A. (2009). Use of the Higuchi's fractal dimension for the analysis of MEG recordings from Alzheimer's disease patients. Med. Eng. Phys. 31, 306-313. doi: 10.1016/j.medengphy.2008.06.010

Good, C. D., Johnsrude, I. S., Ashburner, J., Henson, R. N., Friston, K. J., and Frackowiak, R. S. (2001). A voxel-based morphometric study of ageing in 465 normal adult human brains. Neuroimage 14(1 Pt 1), 21-36. doi: 10.1006/nimg.2001.0786

Grunwald, M., Busse, F., Hensel, A., Kruggel, F., Riedel-Heller, S., Wolf, H., et al. (2001). Correlation between cortical theta activity and hippocampal volumes in health, mild cognitive impairment, and mild dementia. J. Clin. Neurophysiol. 18, 178-184. doi: 10.1097/00004691-200103000-00010

Huang, C., Wahlund, L. O., Dierks, T., Julin, P., Winblad, B., and Jelic, V. (2000). Discrimination of Alzheimer's disease and mild cognitive impairment by equivalent EEG sources: a cross-sectional and longitudinal study. Clin. Neurophysiol. 111, 1961-1967. doi: 10.1016/S1388-2457(00) 00454-5

Hughes, C. P., Berg, L., and Danziger, W. L. (1982). A new clinical scale for the staging of dementia. Br. J. Psychiatry 140, 566-572. doi: 10.1192/bjp.140. 6.566

Jelic, V., Dierks, T., Amberla, K., Almkvist, O., Winblad, B., and Nordberg, A. (1998). Longitudinal changes in quantitative EEG during long-term tacrine treatment of patients with Alzheimer's disease. Neurosci. Lett. 254, 85-88.

Jelic, V., Johansson, S.-E., Almkvist, O., Shigeta, M., Julin, P., Nordberg, A., et al. (2000). Quantitative electroencephalography in mild cognitive impairment: longitudinal changes and possible prediction of Alzheimer's disease. Neurobiol. Aging 21, 533-540. doi: 10.1016/S0197-4580(00) 00153-6

Jelic, V., Shigeta, M., Julin, P., Almkvist, O., Winblad, B., and Wahlund, L. O. (1996). Quantitative electroencephalography power and coherence in Alzheimer's disease and mild cognitive impairment. Dementia 7, 314-323.

Jeong, J. (2004). EEG dynamics in patients with Alzheimer's disease. Clin. Neurophysiol. 115, 1490-1505. doi: 10.1016/j.clinph.2004.01.001

Jurcak, V., Tsuzuki, D., and Dan, I. (2007). 10/20, 10/10, and 10/5 systems revisited: their validity as relative head-surface-based positioning systems. Neuroimage 34, 1600-1611. doi: 10.1016/j.neuroimage.2006.09.024

Kaminski, M., and Blinowska, K. J. (2014). Directed Transfer Function is not influenced by volume conduction-inexpedient pre-processing should be avoided. Front. Comput. Neurosci. 8:61. doi: 10.3389/fncom.2014.00061

Kang, J., Lemaire, H. G., Unterbeck, A., Salbaum, J. M., Masters, C. L., Grzeschik, K. H., et al. (2015). The precursor of Alzheimer's disease amyloid A4 protein resembles a cell-surface receptor. Nature 325, 733-736. doi: 10.1097/00002093198701030-00032

Klimesch, W. (1999). EEG alpha and theta oscillations reflect cognitive and memory performance: a review and analysis. Brain Res. Brain Res. Rev. 29, 169-195.

Klimesch, W., Doppelmayr, M., Schimke, H., and Pachinger, T. (1996). Alpha frequency, reaction time, and the speed of processing information. J. Clin. Neurophysiol. 13, 511-518.

Lawton, M. P., and Brody, E. M. (1969). Assessment of older people: selfmaintaining and instrumental activities of daily living. Gerontologist 9, 179-186. doi: 10.1093/geront/9.3_Part_1.179

Lehmann, C., Koenig, T., Jelic, V., Prichep, L., John, R. E., Wahlund, L.-O., et al. (2007). Application and comparison of classification algorithms for recognition of Alzheimer's disease in electrical brain activity (EEG). J. Neurosci. Methods 161, 342-350. doi: 10.1016/j.jneumeth.2006.10.023

Leite, A. J. B., Scheltens, P., and Barkhof, F. (2004). Pathological aging of the brain: an overview. Top. Magn. Reson. Imaging 15, 369-389.

Leuchter, A. F., Cook, I. A., Newton, T. F., Dunkin, J., Walter, D. O., RosenbergThompson, S., et al. (1993). Regional differences in brain electrical activity in dementia: use of spectral power and spectral ratio measures. Electroencephalogr. Clin. Neurophysiol. 87, 385-393.
Mazziotta, J., Toga, A., Evans, A., Fox, P., Lancaster, J., Zilles, K., et al. (2001) A probabilistic atlas and reference system for the human brain: International Consortium for Brain Mapping (ICBM). Philos. Trans. R. Soc. Lond. B Biol. Sci. 356, 1293-1322. doi: 10.1098/rstb.2001.0915

McKeith, I. G., Dickson, D. W., Lowe, J., Emre, M., O’Brien, J. T., Feldman, H., et al. (2005). Diagnosis and management of dementia with Lewy bodies: third report of the DLB consortium. Neurology 65, 1863-1872. doi: 10.1212/01.wnl.0000187889.17253.b1

McKhann, G., Drachman, D., Folstein, M., Katzman, R., Price, D., and Stadlan, E. M. (1984). Clinical diagnosis of Alzheimer's disease: report of the NINCDSADRDA Work Group under the auspices of Department of Health and Human Services Task Force on Alzheimer's Disease. Neurology 34, 939-944. doi: 10.1212/WNL.34.7.939

Onofrj, M., Thomas, A., Iacono, D., Luciano, A. L., and Di Iorio, A. (2003). The effects of a cholinesterase inhibitor are prominent in patients with fluctuating cognition: a part 3 study of the main mechanism of cholinesterase inhibitors in dementia. Clin. Neuropharmacol. 26, 239-251. doi: 10.1097/00002826200309000-00008

Pascual-Marqui, R. D. (2002). Standardized low resolution brain electromagnetic tomography (sLORETA): technical details. Methods Find Exp. Clin. Pharmacol. 24D, 5-12.

Pascual-Marqui, R. D. (2007a). Discrete, 3D Distributed, Linear Imaging Methods of Electric Neuronal Activity. Part 1: Exact, Zero Error Localization. arXiv:0710.3341 [math-ph]. Available online at: http://arxiv.org/pdf/0710.3341 (Accessed on November 19, 2015).

Pascual-Marqui, R. D. (2007b). Coherence and Phase Synchronization: Generalization to Pairs of Multivariate Time Series, and Removal of Zero-Lag Contributions. arXiv:0706.1776v3 [stat.ME]. Available online at: http://arxiv.org/pdf/0706.1776 (Accessed on November 19, 2015).

Pascual-Marqui, R. D. (2007c). Instantaneous and Lagged Measurements of Linear and Nonlinear Dependence between Groups of Multivariate Time Series: Frequency Decomposition. arXiv:0711.1455 [stat.ME]. Available online at: http://arxiv.org/abs/0711.1455 (Accessed on November 19, 2015).

Pascual-Marqui, R. D., Lehmann, D., Koukkou, M., Kochi, K., Anderer, P., Saletu, B., et al. (2011). Assessing interactions in the brain with exact lowresolution electromagnetic tomography. Philos. Trans. A Math. Phys. Eng. Sci. 369, 3768-3784. doi: 10.1098/rsta.2011.0081

Pascual-Marqui, R. D., Michel, C. M., and Lehmann, D. (1994). Low resolution electromagnetic tomography: a new method for localizing electrical activity in the brain. Int. J. Psychophysiol. 18, 49-65. doi: 10.1016/0167-8760(84) 90014-X

Ponomareva, N. V., Selesneva, N. D., and Jarikov, G. A. (2003). EEG alterations in subjects at high familial risk for Alzheimer's disease. Neuropsychobiology 48, 152-159. doi: 10.1159/000073633

Price, D. L. (2000). Aging of the brain and dementia of the Alzheimer type. Princ. neural Sci. 1149-1168.

Pritchard, W. S., Duke, D. W., Coburn, K. L., Moore, N. C., Tucker, K. A., Jann, M. W., et al. (1994). EEG-based, neural-net predictive classification of Alzheimer's disease versus control subjects is augmented by non-linear EEG measures. Electroencephalogr. Clin. Neurophysiol. 91, 118-130. doi: 10.1016/0013-4694(94)90033-7

Riley, K. P., Snowdon, D. A., Desrosiers, M. F., and Markesbery, W. R. (2005). Early life linguistic ability, late life cognitive function, and neuropathology: findings from the Nun Study. Neurobiol. Aging 26, 341-347. doi: 10.1016/j. neurobiolaging.2004.06.019

Román, G. C., Tatemichi, T. K., Erkinjuntti, T., Cummings, J. L., Masdeu, J. C. Garcia, J. H., et al. (1993). Vascular dementia: diagnostic criteria for research studies. Report of the NINDS-AIREN International Workshop. Neurology 43, 250-260. doi: 10.1212/WNL.43.2.250

Schroeter, M. L., Stein, T., Maslowski, N., and Neumann, J. (2009). Neural correlates of Alzheimer's disease and mild cognitive impairment: a systematic and quantitative meta-analysis involving 1351 patients. Neuroimage 47, 1196-1206. doi: 10.1016/j.neuroimage.2009. 05.037

Shattuck, D. W., Mirza, M., Adisetiyo, V., Hojatkashani, C., Salamon, G., Narr K. L., et al. (2008). Construction of a 3D probabilistic atlas of human cortical structures. Neuroimage 39, 1064-1080. doi: 10.1016/j.neuroimage.2007. 09.031 
Snowdon, D. A. (2003). Healthy Aging and dementia: findings from the nun study. Ann. Int. Med. 139(5 Pt 2), 450-454. doi: 10.7326/0003-4819-139-5_Part_2200309021-00014

The Lund and Manchester Groups (1994). Clinical and neuropathological criteria for frontotemporal dementia. J. Neurol. Neurosurg. Psychiatry 57, 416-418. doi: 10.1136/jnnp.57.4.416

Tyas, S. L., Salazar, J. C., Snowdon, D. A., Desrosiers, M. F., Riley, K. P., Mendiondo, M. S., et al. (2007). Transitions to mild cognitive impairments, dementia, and death: findings from the Nun study. Am. J. Epidemiol. 165, 1231-1238. doi: 10.1093/aje/kwm085

Yesavage, J. A., Brink, T. L., Rose, T. L., Lum, O., Huang, V., Adey, M., et al. (1982). Development and validation of a geriatric depression screening scale: a preliminary report. J. Psychiatr. Res. 17, 37-49.
Conflict of Interest Statement: The authors declare that the research was conducted in the absence of any commercial or financial relationships that could be construed as a potential conflict of interest.

Copyright (C) 2016 Babiloni, Triggiani, Lizio, Cordone, Tattoli, Bevilacqua, Soricelli, Ferri, Nobili, Gesualdo, Millán-Calenti, Buján, Tortelli, Cardinali, Barulli, Giannini, Spagnolo, Armenise, Buenza, Scianatico, Logroscino, Frisoni and del Percio. This is an open-access article distributed under the terms of the Creative Commons Attribution License (CC BY). The use, distribution or reproduction in other forums is permitted, provided the original author(s) or licensor are credited and that the original publication in this journal is cited, in accordance with accepted academic practice. No use, distribution or reproduction is permitted which does not comply with these terms. 